\title{
RIESGO-PAÍS Y TASAS DE DESCUENTO PARA EMPRESAS LATINOAMERICANAS
}

\author{
Jaime Sabal \\ Esade \\ jaime.sabal@esade.edu \\ Aleida Sarmiento \\ Universidad ESAN \\ asarmiento@esan.edu.pe
}

\section{Resumen}

Se identifican las empresas latinoamericanas a las cuales se les puede calcular beta y, por ende, sus tasas de descuento corporativas con un grado aceptable de confiabilidad. La selección se ha basado en las compañías cuyas acciones se cotizan con suficiente liquidez tanto en los mercados bursátiles locales como en la Bolsa de Nueva York. A la fecha de la muestra, 88 empresas han calificado para el cómputo directo de beta. La inestabilidad de los indicadores de liquidez y la propia dinámica del mercado hacen necesario actualizar el análisis con cierta frecuencia.

Palabras clave: beta, beta directo, tasas de descuento en América Latina, riesgo-país, CAPM, liquidez y presencia bursátil.

\begin{abstract}
This paper identifies the Latin American companies for which the beta calculation is possible and consequently their corporate discount rates with an acceptable degree of confidence. The selection is based on companies whose shares are listed with sufficient liquidity in both local stock markets as well as the New York Stock Exchange. At the time of the sample, 88 companies had qualified for the direct beta calculation. The instability of the liquidity indicators and the market dynamics themselves make it necessary to update the analysis often.
\end{abstract}

Key words: beta, direct beta, discount rates in Latin America, country risk, CAPM, liquidity and stock market presence. 


\section{Introducción}

La tasa de descuento aplicable a las inversiones en países emergentes suele ser calculada añadiendo una prima por riesgopaís a la tasa de descuento correspondiente a las inversiones del mismo sector en un país desarrollado. Sin embargo, a pesar de ser una práctica muy generalizada, esta es altamente cuestionable. Lo más correcto sería determinar beta directamente con base en la historia de rendimientos de la empresa de que se trate.

Desafortunadamente, la proporción de empresas con series históricas de rendimientos que a la vez de ser confiables estén disponibles públicamente es baja. Solo aquellas compañías cuyas acciones están listadas en las bolsas de valores tendrían cabida dentro de este grupo privilegiado.

En este trabajo se busca identificar las empresas latinoamericanas a las cuales se les puede calcular beta y, por ende, sus tasas de descuento corporativas, con un grado aceptable de confiabilidad.

El criterio básico ha sido identificar aquellas firmas cuyas acciones se coticen con suficiente liquidez, sea directamente en los mercados bursátiles locales o indirectamente en la Bolsa de Nueva York.

Para juzgar el nivel de liquidez se han utilizado los criterios de frecuencia y volumen de cotización. La muestra definitiva de empresas escogidas deberá cumplir además el requisito de que el cómputo de sus betas con respecto del índice S\&P 500 muestre una confiabilidad mínima aceptable.

\section{Fundamentos}

La práctica más generalizada entre los analistas para determinar la tasa de descuento aplicable a las inversiones en países emergentes es modificar la tasa de descuento indicada por el Capital Asset Pricing Model (CAPM) para inversiones similares en un país desarrollado añadiendo una prima por riesgo-país.

La prima por riesgo-país suele ser estimada como la diferencia entre los rendimientos de los bonos soberanos en el largo plazo (por ejemplo, 10 años) emitidos por un país desarrollado (generalmente Estados Unidos) y el país emergente de que se trate ${ }^{1}$.

Aunque es una práctica que parece razonable a primera vista, una reflexión cuidadosa obliga a cuestionar este procedimiento por las razones que se exponen a continuación (Sabal, 2002).

\subsection{El riesgo crediticio no es el riesgo-país}

El precio de los bonos emitidos por los gobiernos en los mercados financieros internacionales depende fundamentalmente de las expectativas de los inversionistas sobre la eventualidad de incumplimiento en los pagos por parte de dichos gobiernos.

Al incorporar la prima por riesgo-país en la tasa de descuento para una inversión particular se está suponiendo que el riesgo

1. La prima por riesgo-país en ocasiones se determina con base en informes de instituciones internacionales (por ejemplo, Economist Intelligence Unit) o puede verse afectada por otras variables, como el porcentaje de exportaciones de un determinado proyecto o empresa, o la volatilidad del índice bursátil local. 
de incumplimiento del gobierno en cuestión es la medida correcta del riesgo-país propio de dicha inversión, cuando en realidad los riesgos relevantes son más complejos, suelen tener una raíz más social y política que económica y se encuentran íntimamente entrelazados con los otros riesgos que afectan a un proyecto. Por tanto, igualar el riesgo-país al diferencial de rendimientos de los bonos es una práctica incorrecta en la mayoría de los casos.

\section{2. ¿Qué se entiende por «riesgo-país»?}

Es difícil llegar a un consenso sobre el significado de «riesgo-país». El término pareciera referirse a aquellos riesgos que siendo típicos de países en desarrollo pueden afectar negativamente los resultados financieros de una inversión.

Ni siquiera es fácil ponerse de acuerdo sobre cuándo se considera que un país está desarrollado o no. La realidad es que el nivel de desarrollo depende de muchos factores socioculturales y económicos, como el ingreso per cápita, el nivel de educación, la confiabilidad de las instituciones y el marco legal, la estabilidad política y económica, etcétera. De modo que todos los países están ubicados en algún punto intermedio en una gama que va desde un nivel muy bajo hasta un nivel muy alto de desarrollo, y existe un buen número de países que serían difíciles de clasificar en uno u otro grupo (por ejemplo, Taiwán, Corea del Sur, Rusia o Hungría).

Afortunadamente, una definición clara de riesgo-país o del nivel de desarrollo no es necesaria para efectuar las decisiones de inversión en las cuales lo importante es cuantificar cómo las características de un país pueden afectar de manera favorable o desfavorable una decisión de inversión particular.

Cualquiera sea la definición de riesgopaís, este no afecta en la misma forma a todos los proyectos en el mismo país y, por tanto, se debe descartar todo intento de cuantificar el riesgo-país simplemente como una prima sobre la tasa de descuento aplicable a los flujos de caja.

\subsection{El riesgo-país no es totalmente sistemático y es inestable}

Al sumar la prima por riesgo-país a la tasa de libre de riesgo, y por lo tanto a la tasa de descuento (calculada mediante el CAPM), se está suponiendo que el riesgo-país es sistemático (o no diversificable). No obstante, no hay razón para que el riesgo-país tenga una correlación importante con el rendimiento del resto de la economía mundial, por lo que sería posible diversificarlo, al menos en cierta medida.

Como norma general, de ser totalmente sistemático sería correcto sumar la prima por riesgo-país a la tasa de descuento. De ser parcialmente sistemático, una porción debería ser incluida en la tasa de descuento y la otra no. En caso de ser totalmente no sistemático es incorrecto incluir la prima por riesgo-país en la tasa de descuento.

Como respaldo empírico a la posibilidad de que el riesgo-país sea en cierta medida diversificable véase el cuadro 1, que contiene los coeficientes de correlación de los índices bursátiles de los países latinoamericanos más importantes con respecto del índice bursátil S\&P 500 de la Bolsa de Valores de Nueva York (NYSE).

Nótese cómo los coeficientes de correlación más elevados corresponden a economías 


\section{Cuadro 1. Correlación entre los índices bursátiles de América Latina y el S\&P 500* (9 de febrero de 2007)}

\begin{tabular}{lcc}
\hline \multicolumn{1}{c}{ Índice } & País sede & Correlación \\
\hline IPSA & Chile & 0,76 \\
Ibovespa & Brasil & 0,73 \\
Índice de Precios y Cotizaciones & México & 0,68 \\
Merval & Argentina & 0,64 \\
IGBC & Colombia & 0,57 \\
IGBVL & Perú & 0,03 \\
Índice Bursátil Caracas & Venezuela & $-0,08$ \\
\hline
\end{tabular}

Fuente: Economática.

* Se ha tomado cotizaciones semanales para un año, expresadas en dólares.

relativamente más desarrolladas e integradas a los mercados internacionales.

Por tanto, pareciera que en la medida en que la economía de un país esté menos desarrollada y/o se encuentre menos integrada a los mercados mundiales será más diversificable el riesgo asociado a estos países y la tasa de descuento se verá menos afectada por un riesgo-país particular ${ }^{2}$.

El procedimiento más consistente con los principios de la teoría financiera moderna y en particular con el CAPM sería estimar directamente el beta de cada empresa en particular con respecto de un índice representativo de la cartera de mercado, generalmente el S\&P 500. De esta forma, la porción sistemática del riesgo país asociada con el proyecto analizado se reflejaría directamente en el cómputo de beta.

Desgraciadamente, son solo una minoría las empresas de países emergentes que están listadas en bolsa y cuyas cotizaciones

2. Esta conclusión no es del todo confiable puesto que las bolsas de valores de los países emergentes generalmente están lejos de incluir un grupo de empresas suficientemente representativo de la actividad económica general de cada nación, por lo que las correlaciones del cuadro 1 no reflejan en forma muy precisa la relación entre las economías de cada país y el S\&P 500, sino que sirven como punto de referencia. son lo suficientemente líquidas para arrojar resultados confiables.

\section{Criterios para la identificación de las empresas}

El criterio básico es identificar aquellas empresas cuyas acciones se coticen en los mercados bursátiles con liquidez suficiente para permitir que sus beta sean computados de manera confiable. Para juzgar el nivel de liquidez se considerarán los criterios de frecuencia, volumen y rotación.

\section{a) Frecuencia}

La cotización en bolsa tiene que haber sido frecuente; es decir, la proporción de días de transacción sobre el número total de días hábiles del año deberá ser lo más alta posible. Lo ideal es que la acción sea transada todos los días. En este caso se habla de un índice de presencia del ciento por ciento.

\section{b) Volumen}

El volumen transado debe ser significativo. En este trabajo de establece como criterio mínimo de liquidez un volumen diario negociado de 500 mil dólares estadounidenses $^{3}$.

3. Basado en la recomendación de Pristine Capital Holdings, Inc. (disponible en <www.pristine.com>). 


\section{c) Rotación}

Las acciones negociadas deberán representar un porcentaje elevado del número de acciones en circulación. El índice de rotación se define como:

(Número de acciones negociadas / Número de acciones emitidas)

No obstante, la concentración accionarial afecta sensiblemente este indicador, pues cuanto mayor sea el porcentaje de acciones pertenecientes al grupo que mantiene el control de la empresa menor será el índice de rotación. De modo que aunque las acciones fuera del grupo de control fuesen transadas muy activamente, el índice de rotación seguiría siendo relativamente bajo. Con el propósito de profundizar en esta dificultad se ha calculado la concentración accionarial, definida como el porcentaje de acciones en manos de los cinco primeros accionistas de las empresas cotizadas en las bolsas de valores de los países seleccionados.

Por otra parte, muchas de las empresas latinoamericanas dentro del universo que se busca identificar han emitido ADR en algún mercado financiero desarrollado. Por tanto, el cómputo de sus betas puede hacerse directamente a través de las acciones emitidas en los mercados locales o a través de los ADR en cuestión ${ }^{4}$.

En el cuadro 2 se presentan los porcentajes del accionariado de las empresas latinoamericanas que pertenecen a los primeros cinco accionistas mayoritarios, también se incluyen las empresas que han emitido ADR en los mercados financieros desarrollados.

\section{Cuadro 2. Empresas de América Latina cotizadas en bolsa (local o como ADR) con parte de sus acciones en manos de los cinco primeros accionistas ${ }^{5}$ (9 de febrero de 2007)}

\begin{tabular}{cccc}
\hline & $\begin{array}{c}\text { Número de } \\
\text { empresas }\end{array}$ & Porcentaje & $\begin{array}{c}\text { Porcentaje } \\
\text { acumulado }\end{array}$ \\
\hline $100 \%$ & 40 & 5,24 & 5,24 \\
$90-99 \%$ & 206 & 27,00 & 32,24 \\
$80-89 \%$ & 133 & 17,43 & 49,67 \\
$70-79 \%$ & 99 & 12,98 & 62,65 \\
$60-69 \%$ & 84 & 11,01 & 73,66 \\
$50-59 \%$ & 81 & 10.62 & 84,27 \\
$40-49 \%$ & 49 & 6,42 & 90,69 \\
$30-39 \%$ & 50 & 6,55 & 97,25 \\
$20-29 \%$ & 13 & 1,70 & 98,95 \\
Difuminado & 8 & 1,05 & 100,00 \\
\hline Total & $\mathbf{7 6 3}$ & $\mathbf{1 0 0 , 0 0}$ & \\
\hline
\end{tabular}

Fuente: Economática.

4. Los American Depositary Receipts (ADR) son documentos que se emiten a cambio de acciones de una corporación no estadounidense que se encuentran en la bóveda de algún banco y otorgan a sus propietarios todos los derechos sobre los dividendos y las ganancias de capital correspondientes. También existen los Global Depositary Receipts (GDR), los cuales se diferencian de los ADR en que pueden ser negociados en bolsas de valores fuera de Estados Unidos (por ejemplo, en la Bolsa de Madrid).

5. Se considera solo aquellas empresas que incluían el dato del accionariado. Según la base Economática, de un total de 1025 empresas latinoamericanas que se encuentran activas y han emitido acciones o ADR, 262 no brindan el dato de su accionariado. 
Como era de esperarse, dado que la gran mayoría de las empresas de América Latina tienen accionariados poco difundidos o son empresas familiares, es relativamente bajo el porcentaje de sus acciones que son activamente negociadas en los mercados de valores. Por esta razón, concluimos que el índice de rotación no tiene cabida como criterio de selección, por lo que será descartado en este trabajo.

\section{Identificación con base en mercados locales}

A la fecha de corte de este estudio (9 de febrero de 2007), un total de 1439 empresas latinoamericanas habían emitido acciones en sus bolsas locales. De ellas, 1019 se mantenían activas y emitían en conjunto 1462 diferentes acciones. El objetivo es identificar cuáles pueden ser consideradas acciones líquidas.

\subsection{Criterio de frecuencia}

En el gráfico 1 se aplica un filtro para un índice de presencia del ciento por ciento para periodos de una a doce semanas, se observa así una tendencia decreciente en el número de empresas que lo superan: 489 acciones han tenido un $100 \%$ de presencia durante una semana pero solamente 311 la han tenido a lo largo de doce semanas.

Para obtener una muestra que evite distorsiones por compras extraordinarias durante las últimas semanas, se ha restringido la selección a las 380 acciones con $100 \%$ de presencia durante las últimas cuatro semanas.

\subsection{Criterio de volumen}

Luego de aplicar un filtro por el volumen negociado con un corte de 500 mil dólares estadounidenses a las 380 acciones resul-

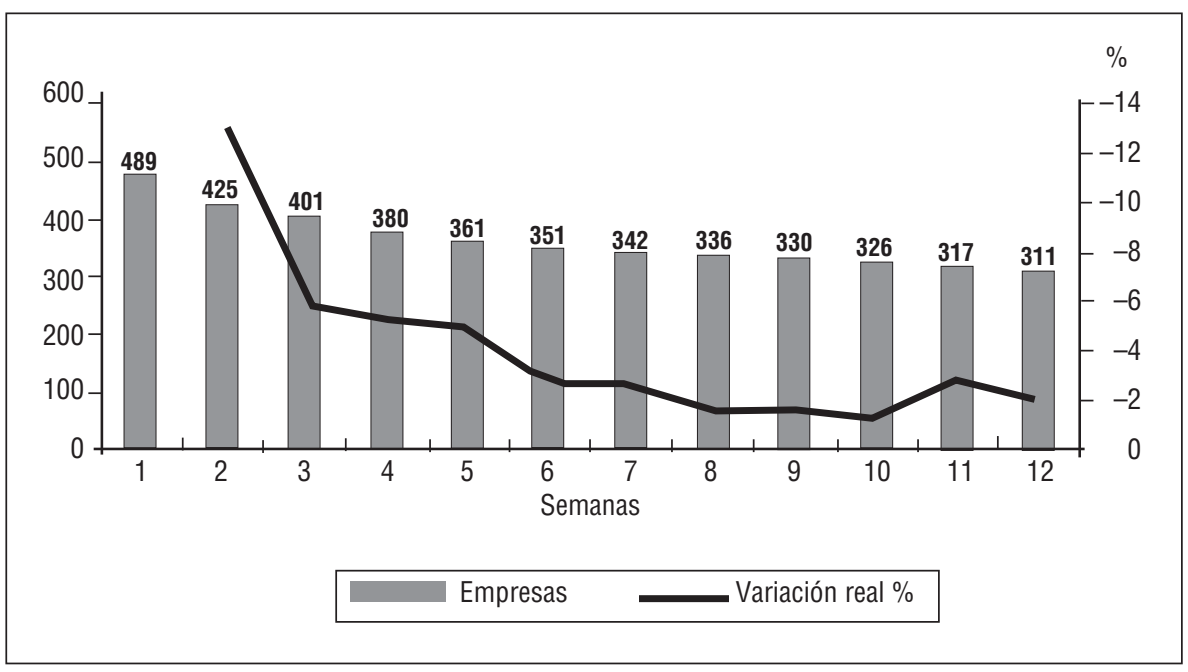

Fuente: Economática.

\section{Gráfico 1}

Resultados en la muestra seleccionada del filtro de presencia al 100\% (por semanas, al 9 de febrero de 2007) 
tantes, quedó un total de 211 acciones emitidas por 202 empresas. En el cuadro 3 se presentan los sectores y los países a los cuales pertenecen las acciones y las empresas en cuestión. En el anexo 1 aparece el listado con todas las empresas de este grupo que han superado los filtros de liquidez.

Llama la atención que luego de aplicar el corte no aparece ninguna empresa de Venezuela. Un análisis similar realizado en octubre del año 2006 mantenía una empresa venezolana en la lista. Este hecho, además de ilustrar la escasa estabilidad de los indicadores de liquidez, enfatiza la necesidad de actualizar los resultados de esta investigación con frecuencia.

Se puede concluir que de acuerdo con los criterios utilizados un total de $202 \mathrm{em}$ presas de la región cuentan con acciones cotizadas que tienen liquidez suficiente en sus mercados locales.

No obstante, como se verá más adelante, desafortunadamente el cómputo de beta no arroja suficiente confiabilidad para la mayoría de estas empresas; de forma que solo para un número reducido de ellas se justificará la aplicación del CAPM.

Cuadro 3. Características de los emisores locales en América Latina* (después de aplicar filtros por liquidez y volumen)

\begin{tabular}{|c|c|c|c|c|c|c|c|c|c|c|c|c|c|c|}
\hline & \multicolumn{2}{|c|}{ ARGENTINA } & \multicolumn{2}{|c|}{ BRASIL } & \multicolumn{2}{|c|}{ CHILE } & \multicolumn{2}{|c|}{ COLOMBIA } & \multicolumn{2}{|c|}{ MÉXICO } & \multicolumn{2}{|c|}{ PERÚ } & \multicolumn{2}{|c|}{ TOTAL } \\
\hline & Acc. & Emp. & Acc. & Emp. & Acc. & Emp. & Acc. & Emp. & Acc. & Emp. & Acc. & Emp. & Acc. & Emp. \\
\hline Agro y Pesca & - & - & - & - & 1 & 1 & - & - & - & - & - & - & 1 & 1 \\
\hline \multicolumn{15}{|l|}{ Alimentos y } \\
\hline Bebidas & 1 & 1 & 6 & 5 & 3 & 3 & - & - & 7 & 7 & - & - & 17 & 16 \\
\hline Comercio & - & - & 5 & 5 & 5 & 5 & - & - & 4 & 4 & - & - & 14 & 14 \\
\hline Construcción & - & - & 6 & 6 & - & - & - & - & 9 & 9 & - & - & 15 & 15 \\
\hline Electrónica & - & - & 1 & 1 & - & - & - & - & - & - & - & - & 1 & 1 \\
\hline Energía eléctrica & - & - & 15 & 14 & 5 & 5 & 1 & 1 & - & - & - & - & 21 & 20 \\
\hline \multicolumn{15}{|l|}{ Finanzas y } \\
\hline Seguros & 1 & 1 & 8 & 7 & 7 & 7 & 4 & 4 & 2 & 2 & 1 & 1 & 23 & 22 \\
\hline \multicolumn{15}{|l|}{ Maquinaria } \\
\hline industrial & - & - & 1 & 1 & - & - & - & - & - & - & - & - & 1 & 1 \\
\hline \multicolumn{15}{|l|}{ Minerales no } \\
\hline metálicos & - & - & 1 & 1 & 1 & 1 & 1 & 1 & 1 & 1 & - & - & 4 & 4 \\
\hline Minería & - & - & 2 & 1 & 1 & 1 & - & - & 2 & 2 & 6 & 6 & 11 & 10 \\
\hline Otros & 2 & 2 & 15 & 15 & 2 & 2 & - & - & 3 & 3 & - & - & 22 & 22 \\
\hline Papel y Celulosa & - & - & 4 & 4 & 1 & 1 & - & - & 1 & 1 & - & - & 6 & 6 \\
\hline Petróleo y Gas & - & - & 4 & 3 & 1 & 1 & - & - & - & - & - & - & 5 & 4 \\
\hline Química & - & - & 5 & 5 & - & - & - & - & 1 & 1 & - & - & 6 & 6 \\
\hline \multicolumn{15}{|l|}{ Siderurgia y } \\
\hline Metalurgia & 2 & 2 & 12 & 11 & 3 & 3 & - & - & 3 & 3 & 1 & 1 & 21 & 20 \\
\hline Software y Datos & - & - & 2 & 2 & 1 & 1 & - & - & - & - & - & - & 3 & 3 \\
\hline Telecomunicación & 1 & 1 & 11 & 8 & 2 & 2 & - & - & 3 & 3 & - & - & 17 & 14 \\
\hline Textil & - & - & 2 & 2 & - & - & 1 & 1 & - & - & - & - & 3 & 3 \\
\hline \multicolumn{15}{|l|}{ Transporte y } \\
\hline Servicios & - & - & 8 & 8 & 2 & 2 & - & - & 4 & 4 & - & - & 14 & 14 \\
\hline \multicolumn{15}{|l|}{ Vehículos y } \\
\hline Piezas & - & - & 6 & 6 & - & - & - & - & - & - & - & - & 6 & 6 \\
\hline Total & 7 & 7 & 114 & 105 & 35 & 35 & 7 & 7 & 40 & 40 & 8 & 8 & 211 & 202 \\
\hline
\end{tabular}




\section{Identificación con base en los ADR}

A la fecha de corte, un total de 113 ADR había sido emitido en el NYSE por 106 empresas de América Latina. En el cuadro 4 se clasifica este grupo de empresas emisoras según sector económico y país. El listado por empresa se presenta en el anexo 2.

A la fecha de corte seguían vigentes 95 ADR emitidos por 90 empresas, pues los siguientes habían salido del mercado:
- De ARgentina: Tenaris Siderca S. A. (siderúrgica).

- DE BRASIL: Perdigao S. A. (alimentos), Tco Celular Participacoes S. A. (telecomunicaciones -telecom.), Tele Leste Celular Participacoes S. A. (telecom.), Tele Nordeste Celular Participacoes S A. (telecom.), Tele Sudeste Celular Participacoes S. A. (telecom.), Telecomunicacoes Brasileiras S. A. (Telebras -telecom.) y Emp. Brasileira Aeronautica S. A. (Embraer-vehículos).

Cuadro 4. Número de empresas de América Latina que han emitido ADR en el NYSE

\begin{tabular}{|c|c|c|c|c|c|c|c|c|c|c|c|c|c|c|c|c|}
\hline & \multicolumn{2}{|c|}{ ARGENTINA } & \multicolumn{2}{|c|}{ BRASIL } & \multicolumn{2}{|c|}{ CHILE } & \multicolumn{2}{|c|}{ COLOMBIA } & \multicolumn{2}{|c|}{ MÉXICO } & \multicolumn{2}{|c|}{ PERÚ } & \multicolumn{2}{|c|}{ VENEZUELA } & \multicolumn{2}{|c|}{ TOTAL } \\
\hline & ADR & Emp. & ADR & Emp. & $\overline{A D R}$ & $\overline{\text { Emp. }}$ & ADR & Emp. & $\overline{A D R}$ & Emp. & ADR & Emp. & ADR & Emp. & ADR & Emp. \\
\hline Agro y Pesca & - & - & - & - & 2 & 2 & - & - & 2 & 2 & - & - & - & - & 4 & 4 \\
\hline Alimentos y & & & & & & & & & & & & & & & & \\
\hline $\begin{array}{l}\text { Bebic } \\
\text { Comer }\end{array}$ & $\begin{array}{l}1 \\
-\end{array}$ & $\begin{array}{l}1 \\
-\end{array}$ & $\begin{array}{l}5 \\
1\end{array}$ & $\begin{array}{l}3 \\
1\end{array}$ & $\begin{array}{l}4 \\
2\end{array}$ & $\begin{array}{l}3 \\
2\end{array}$ & - & - & $\begin{array}{l}5 \\
3\end{array}$ & $\begin{array}{l}5 \\
3\end{array}$ & - & $\begin{array}{l}- \\
-\end{array}$ & $\begin{array}{l}- \\
-\end{array}$ & - & $\begin{array}{r}15 \\
6\end{array}$ & $\begin{array}{r}12 \\
6\end{array}$ \\
\hline Construcción & - & - & - & - & - & - & - & - & 2 & 2 & - & - & - & - & 2 & 2 \\
\hline $\begin{array}{r}\text { eléctrica } \\
\text { Finanzas y }\end{array}$ & - & - & 3 & 3 & 2 & 2 & - & - & - & - & - & - & - & - & 5 & 5 \\
\hline Seg & 2 & 2 & 3 & 3 & 4 & 4 & 1 & 1 & - & - & 2 & 2 & - & - & 11 & 11 \\
\hline $\begin{array}{l}\text { Fondos } \\
\text { Maquina }\end{array}$ & - & - & - & - & 2 & 2 & - & - & - & - & - & - & - & - & 2 & 2 \\
\hline $\begin{array}{l}\text { industrial } \\
\text { Minerales no }\end{array}$ & - & - & - & - & - & - & - & - & 1 & 1 & - & - & - & - & 1 & 1 \\
\hline metálicos & - & - & - & - & 1 & 1 & - & - & 3 & 3 & - & - & - & - & 4 & 4 \\
\hline Minería & - & - & 2 & 2 & 1 & 1 & - & - & - & - & 1 & 1 & - & - & 5 & 3 \\
\hline $\begin{array}{l}\text { Otros } \\
\text { Papel y }\end{array}$ & 3 & 3 & 1 & 1 & 1 & 1 & - & - & 3 & 3 & 1 & 1 & - & - & 9 & 9 \\
\hline $\begin{array}{l}\text { Celulosa } \\
\text { etróleo y }\end{array}$ & - & - & 2 & 2 & - & - & - & - & 1 & 1 & - & - & - & - & 3 & 3 \\
\hline Gas & 3 & 3 & 2 & 1 & - & - & - & - & - & - & - & - & - & - & 5 & 4 \\
\hline $\begin{array}{l}\text { Química } \\
\text { Siderurgia y }\end{array}$ & - & - & 2 & 2 & 1 & 1 & - & - & - & - & - & - & - & - & 3 & 3 \\
\hline $\begin{array}{l}\text { Metalurgia } \\
\text { Telecomuni- }\end{array}$ & 3 & 3 & 2 & 2 & 1 & 1 & - & - & 1 & 1 & - & - & - & - & 7 & 7 \\
\hline $\begin{array}{l}\text { cación } \\
\text { Transporte y }\end{array}$ & 2 & 2 & 14 & 14 & 2 & 2 & - & - & 3 & 3 & 1 & 1 & 1 & 1 & 23 & 23 \\
\hline $\begin{array}{c}\text { Servicios } \\
\text { Vehículos y }\end{array}$ & - & - & 2 & 2 & 1 & 1 & - & - & 3 & 3 & - & - & - & - & 6 & 6 \\
\hline Piezas & - & - & 2 & 1 & - & - & - & - & - & - & - & - & - & - & 2 & 1 \\
\hline Total & 14 & 14 & 41 & 37 & 24 & 23 & 1 & 1 & 27 & 27 & 5 & 5 & 1 & 1 & 113 & 106 \\
\hline
\end{tabular}

Fuente: Economática. 
- DE CHILE: Edwards (finanzas), Exmasisa (madera), Labchile (química) y Telex (telecom.).

- DE MÉXICO: Savia, S. A. de C. V. (agro y pesca), Panamco México, S.A. de C.V. (alimentos y bebidas), Pepsi Gemex, S. A. de C. V. (alimentos y bebidas) y Desc S. A. (maquinarias industriales).

- DEL PERÚ: Banco Wiese (ahora Scotiabank Perú -finanzas) y Telefónica del Perú S. A. A. (telecom.).

A continuación se aplicarán los criterios de liquidez a este grupo de 90 empresas.

\subsection{Criterio de frecuencia}

El primer filtro es la frecuencia de negociación medida a través de la presencia en bolsa durante las últimas cuatro semanas. Al igual que se hizo con las acciones cotizadas localmente, el corte se establece en una presencia del 100\% todos los días del último mes. La aplicación de este filtro deja 75 empresas listadas activas en bolsa, las cuales se presentan en el cuadro 5 clasificadas por sector y país.

\subsection{Criterio de volumen}

La lista se reduce a 60 empresas cuando se aplica el criterio de un volumen diario mínimo negociado de 500 mil dólares estadounidenses. Estas empresas aparecen en el cuadro 6 según sector y país. La lista de las empresas se presenta en el anexo 3.

\section{Plazo y frecuencia óptimos de la data}

El que una determinada acción o un ADR cuente con suficiente liquidez no basta para garantizar un cómputo confiable para su beta correspondiente. Además es necesario que exista un mínimo de confiabilidad en la

Cuadro 5. Empresas con ADR que pasan el filtro de frecuencia

\begin{tabular}{lcccccccc}
\hline & Argentina & Brasil & Chile & Colombia & México & Perú & Venezuela & Total \\
\hline Agro y Pesca & - & - & 1 & - & 1 & - & - & 2 \\
Alimentos y Bebidas & 1 & 3 & 2 & - & 2 & - & - & 8 \\
Comercio & - & 1 & 1 & - & - & - & - & 2 \\
Construcción & - & - & - & - & 2 & - & - & 2 \\
Energía eléctrica & - & 3 & 2 & - & - & - & - & 5 \\
Finanzas y Seguros & 2 & 3 & 2 & 1 & - & - & - & 8 \\
Fondos & - & - & 1 & - & - & - & - & 1 \\
Maquinaria industrial & - & - & - & - & - & - & - & 0 \\
Minerales no metálicos & - & - & - & - & 2 & 1 & - & 3 \\
Minería & - & 1 & 1 & - & - & - & - & 2 \\
Otros & 3 & 1 & - & - & 2 & 1 & - & 7 \\
Papel y Celulosa & - & 2 & - & - & - & - & - & 2 \\
Petróleo y Gas & 3 & 1 & - & - & - & - & - & 4 \\
Química & - & 2 & - & - & - & - & - & 2 \\
Siderurgia y Metalurgia & 2 & 2 & 1 & - & - & - & - & 5 \\
Telecomunicación & 2 & 9 & 1 & - & 2 & - & 1 & 15 \\
Transporte y Servicios & - & 2 & 1 & - & 3 & - & - & 6 \\
Vehículos y Piezas & - & 1 & - & - & - & - & - & 1 \\
\hline Total & $\mathbf{1 3}$ & $\mathbf{3 1}$ & $\mathbf{1 3}$ & $\mathbf{1}$ & $\mathbf{1 4}$ & $\mathbf{2}$ & $\mathbf{1}$ & $\mathbf{7 5}$ \\
\hline Fuente: Economática. & & & & & & & &
\end{tabular}


Cuadro 6. Empresas con ADR que pasan el filtro por frecuencia y volumen

\begin{tabular}{lcccccccc}
\hline & Argentina & Brasil & Chile & Colombia & México & Perú & Venezuela & Total \\
\hline Agro y Pesca & - & - & 1 & - & - & - & - & 1 \\
Alimentos y Bebidas & 1 & 3 & 1 & - & 2 & - & - & 7 \\
Comercio & - & 1 & - & - & - & - & - & 1 \\
Construcción & - & - & - & - & 1 & - & - & 1 \\
Energía eléctrica & - & 3 & 2 & - & - & - & - & 5 \\
Finanzas y Seguros & 2 & 3 & 2 & 1 & - & - & - & 8 \\
Minerales no metálicos & - & - & - & - & 1 & - & - & 1 \\
Minería & - & 1 & 1 & - & - & 1 & - & 3 \\
Otros & 2 & 1 & - & - & 1 & 1 & - & 5 \\
Papel y Celulosa & - & 2 & - & - & - & - & - & 2 \\
Petróleo y Gas & - & 1 & - & - & - & - & - & 1 \\
Química & - & 1 & - & - & - & - & - & 1 \\
Siderurgia y Metalurgia & 2 & 2 & - & - & - & - & - & 4 \\
Telecomunicación & 2 & 7 & 1 & - & 2 & - & 1 & 13 \\
Transporte y Servicios & - & 2 & 1 & - & 3 & - & - & 6 \\
Vehículos y Piezas & - & 1 & - & - & - & - & - & 1 \\
\hline Total & $\mathbf{9}$ & $\mathbf{2 8}$ & $\mathbf{9}$ & $\mathbf{1}$ & $\mathbf{1 0}$ & $\mathbf{2}$ & $\mathbf{1}$ & $\mathbf{6 0}$ \\
\hline
\end{tabular}

Fuente: Economática.

relación entre las series de rendimientos del instrumento respectivo y el índice accionario de referencia.

El índice accionario que se utilizará como referente en este trabajo es el S\&P 500, aquel empleado con mayor frecuencia por los analistas del mercado de valores estadounidense. El nivel de confiabilidad de la relación se medirá a través del coeficiente de determinación $\left(\mathrm{R}^{2}\right)$.

En primer lugar es necesario detectar aquellas escalas de tiempo que arrojan un mejor coeficiente de determinación, pues los datos acerca de los rendimientos históricos pueden tener una base diaria, semanal, mensual, etc. y las series se pueden extender uno, dos, tres o más años hacia el pasado.

En este trabajo se calcularán los coeficientes de determinación con rendimientos diarios, semanales y mensuales para lapsos de uno, tres y cinco años. A partir de los resultados obtenidos se elegirá la combinación óptima de escalas de tiempo.
La comparación de los datos se hará mediante funciones lógicas (ver anexo 5) que permitirán determinar qué $\mathrm{R}^{2}$ (semanal, mensual o anual para uno, tres o cinco años) tiene mayor representatividad.

La lista de las empresas y sus nueve coeficientes (mensual, semanal y diario a uno, tres y cinco años) se presenta en el anexo 4 . De su análisis se concluye que la estrategia óptima es utilizar $\mathrm{R}^{2}$ medidos semanalmente en el plazo de un año.

\section{Nivel de confiabilidad}

El siguiente paso es establecer cuál debería ser el coeficiente de determinación mínimo para asegurar que efectivamente exista una relación lineal entre el instrumento y el índice. Si bien un corte al 0,9 (90\%) o más aun al 0,99 (99\%) se consideraría altamente representativo estadísticamente, se puede observar que en la práctica este índice no lo cumplen ni las acciones que forman parte del índice S\&P 500, las cuales en promedio 
alcanzan alrededor del 20\%. Por tanto, esperar que los instrumentos latinoamericanos cumplan con esta representatividad sería irreal, por lo que es preferible buscar que cuenten con un $\mathrm{R}^{2}$ similar al de aquellas acciones estadounidenses razonablemente líquidas.
En el cuadro 7 se presenta la lista de acciones de aquellas empresas que tienen participación en el índice S\&P $500^{6}$ y sus $\mathrm{R}^{2}$.

Se observa que el $\mathrm{R}^{2}$ más elevado es de $64 \%$, el valor promedio es $23 \%$ y la mediana es $21 \%$. Se establecerá entonces

\section{Cuadro 7. Acciones de Estados Unidos y sus $R^{2}$ respecto del S\&P 500 (9 de febrero de 2007)}

\begin{tabular}{|c|c|c|c|}
\hline Razón social & $\mathbf{R}^{2}$ & Razón social & $\mathbf{R}^{2}$ \\
\hline 1. Bemis Co Inc & 0,64 & 36. Ameriprise Financial Inc & 0,49 \\
\hline 2. Air Products y Chemicals Inc /De/ & 0,64 & 37. Merrill Lynch y Co Inc & 0,49 \\
\hline 3. Dover Corp & 0,64 & 38. Mbia Inc & 0,49 \\
\hline 4. Praxair Inc & 0,49 & 39. Becton Dickinson y Co & 0,49 \\
\hline 5. Stanley Works & 0,49 & 40. Csx Corp & 0,49 \\
\hline 6. Kb Home & 0,49 & 41. Leggett y Platt Inc & 0,49 \\
\hline 7. State Street Corp & 0,49 & 42. United Technologies Corp /De/ & 0,49 \\
\hline 8. Masco Corp /De/ & 0,49 & 43. United States Steel Corp & 0,49 \\
\hline 9. Honeywell International Inc & 0,49 & 44. Textron Inc & 0,49 \\
\hline 10. Morgan Stanley & 0,49 & 45. Pactiv Corp & 0,49 \\
\hline 11. Union Pacific Corp & 0,49 & 46. Bear Stearns Companies Inc & 0,49 \\
\hline 12. Sempra Energy & 0,49 & 47. Lennar Corp /New/ & 0,36 \\
\hline 13. Parker Hannifin Corp & 0,49 & 48. International Paper Co /New/ & 0,36 \\
\hline 14. Lehman Brothers Holdings Inc & 0,49 & 49. Dupont E I De Nemours y Co & 0,36 \\
\hline 15. Goodrich Corp & 0,49 & 50. Illinois Tool Works Inc & 0,36 \\
\hline 16. ITT Corp & 0,49 & 51. Snap On Inc & 0,36 \\
\hline 17. American Express Co & 0,49 & 52. Weyerhaeuser Co & 0,36 \\
\hline 18. Tyco International Ltd /Ber/ & 0,49 & 53. American Standard Companies Inc & 0,36 \\
\hline 19. Danaher Corp /De/ & 0,49 & 54. International Business Machines Corp & 0,36 \\
\hline 20. Deere y Co & 0,49 & 55. Rohm y Haas Co & 0,36 \\
\hline 21. Cooper Industries Ltd & 0,49 & 56. Federated Department Stores Inc /De/ & 0,36 \\
\hline 22. Goldman Sachs Group Inc/ & 0,49 & 57. Archstone Smith Trust & 0,36 \\
\hline 23. Xerox Corp & 0,49 & 58. Ppg Industries Inc & 0,36 \\
\hline 24. Teradyne, Inc & 0,49 & 59. Rockwell Collins Inc & 0,36 \\
\hline 25. Horton D R Inc /De/ & 0,49 & 60. Ball Corp & 0,36 \\
\hline 26. Centex Corp & 0,49 & 61. Brunswick Corp & 0,36 \\
\hline 27. Thermo Electron Corp & 0,49 & 62. Home Depot Inc & 0,36 \\
\hline 28. Vulcan Materials Co & 0,49 & 63. Lincoln National Corp & 0,36 \\
\hline 29. J P Morgan Chase y Co & 0,49 & 64. Cb Richard Ellis Group Inc & 0,36 \\
\hline 30. Pulte Homes Inc/Mi/ & 0,49 & 65. Pall Corp & 0,36 \\
\hline 31. Fortune Brands Inc & 0,49 & 66. Lowes Companies Inc & 0,36 \\
\hline 32. Franklin Resources Inc & 0,49 & 67. Electronic Data Systems Corp /De/ & 0,36 \\
\hline 33. Burlington Northern Santa Fe Corp & 0,49 & 68. Carnival Corp & 0,36 \\
\hline 34. Genuine Parts Co & 0,49 & 69. General Electric Co & 0,36 \\
\hline 35. Agilent Technologies Inc & 0,49 & 70. Mellon Financial Corp & 0,36 \\
\hline
\end{tabular}

6. Para el cálculo de este coeficiente se han tomado valores semanales del último año, de acuerdo con las conclusiones de la sección anterior. 


\begin{tabular}{|c|c|c|c|}
\hline Razón social & $\mathbf{R}^{2}$ & Razón social & $\mathbf{R}^{2}$ \\
\hline 71. Principal Financial Group Inc & 0,36 & 125. Citizens Communications Co & 0,25 \\
\hline 72. Fedex Corp & 0,36 & 126. Tektronix Inc & 0,25 \\
\hline 73. Eastman Chemical Co & 0,36 & 127. Rowan Companies Inc & 0,25 \\
\hline 74. Freescale Semiconductor Inc & 0,36 & 128. Public Storage Inc /Ca & 0,25 \\
\hline 75. Simon Property Group Inc /De/ & 0,36 & 129. Ryder System Inc & 0,25 \\
\hline 76. Eaton Corp & 0,36 & 130. PgyE Corp & 0,25 \\
\hline 77. MyT Bank Corp & 0,36 & 131. Rockwell Automation Inc & 0,25 \\
\hline 78. Wachovia Corp New & 0,36 & 132. Autonation Inc/FI & 0,25 \\
\hline 79. Ace Ltd & 0,36 & 133. Peabody Energy Corp & 0,25 \\
\hline 80. Duke Energy Corp & 0,36 & 134. Waters Corp /De/ & 0,25 \\
\hline 81. Plum Creek Timber Co Inc & 0,36 & 135. Grainger W W Inc & 0,25 \\
\hline 82. Norfolk Southern Corp & 0,36 & 136. Eog Resources Inc & 0,25 \\
\hline 83. Allegheny Technologies Inc & 0,36 & 137. Fluor Corp & 0,25 \\
\hline 84. American International Group Inc & 0,36 & 138. Verizon Communications Inc & 0,25 \\
\hline 85. National Semiconductor Corp & 0,36 & 139. Solectron Corp & 0,25 \\
\hline 86. Teco Energy Inc & 0,36 & 140. Circuit City Stores Inc & 0,25 \\
\hline 87. Half Robert International Inc /De/ & 0,36 & 141. Big Lots Inc & 0,25 \\
\hline 88. Clorox Co /De/ & 0,36 & 142. Metlife Inc & 0,25 \\
\hline 89. Newell Rubbermaid Inc & 0,36 & 143. Bj Services Co & 0,25 \\
\hline 90. Marriott International Inc /Md/ & 0,36 & 144. Federated Investors Inc /Pa/ & 0,25 \\
\hline 91. Millipore Corp /Ma & 0,36 & 145. Nordstrom Inc & 0,25 \\
\hline 92. Fidelity National Information Services, Inc. & 0,36 & 146. Claiborne Liz Inc & 0,25 \\
\hline 93. Hartford Financial Services Group Inc/De & 0,36 & 147. Heinz H J Co & 0,25 \\
\hline 94. Tiffany y Co & 0,36 & 148. Allied Waste Industries Inc & 0,25 \\
\hline 95. Prologis & 0,36 & 149. Pfizer Inc & 0,25 \\
\hline 96. Loews Corp & 0,36 & 150. Equity Residential & 0,25 \\
\hline 97. Hewlett Packard Co & 0,36 & 151. Williams Companies Inc & 0,25 \\
\hline 98. International Game Technology & 0,36 & 152. Mgic Investment Corp & 0,25 \\
\hline 99. Progress Energy Inc & 0,36 & 153. Bank Of New York Co Inc & 0,25 \\
\hline 100. Waste Management Inc & 0,36 & 154. Monsanto Co /New/ & 0,25 \\
\hline 101. Meadwestvaco Corp & 0,36 & 155. Harley Davidson Inc & 0,25 \\
\hline 102. Emerson Electric $\mathrm{Co}$ & 0,36 & 156. Citigroup Inc & 0,25 \\
\hline 103. Kimco Realty Corp & 0,36 & 157. Moodys Corp /De/ & 0,25 \\
\hline 104. Coach Inc & 0,36 & 158. Family Dollar Stores Inc & 0,25 \\
\hline 105. Eastman Kodak Co & 0,36 & 159. Torchmark Corp & 0,25 \\
\hline 106. Mylan Laboratories Inc & 0,36 & 160. Darden Restaurants Inc & 0,25 \\
\hline 107. Best Buy Co Inc & 0,36 & 161. Vornado Realty Trust & 0,25 \\
\hline 108. Cummins Inc & 0,36 & 162. Target Corp & 0,25 \\
\hline 109. 3M Co & 0,36 & 163. Allstate Corp & 0,25 \\
\hline 110. Temple Inland Inc & 0,36 & 164. Dow Chemical Co /De/ & 0,25 \\
\hline 111. Caterpillar Inc & 0,36 & 165. Boston Properties Inc & 0,25 \\
\hline 112. Nicor Inc & 0,36 & 166. Apache Corp & 0,25 \\
\hline 113. Hercules Inc & 0,36 & 167. Raytheon Co/ & 0,25 \\
\hline 114. Schering Plough Corp & 0,36 & 168. Amerisourcebergen Corp & 0,25 \\
\hline 115. Cbs Corp & 0,36 & 169. Apartment Investment y Management $\mathrm{Co}_{0}$ & 0,25 \\
\hline 116. Texas Instruments Inc & 0,36 & 170. Allegheny Energy, Inc & 0,25 \\
\hline 117. Whirlpool Corp /De/ & 0,36 & 171. Janus Capital Group Inc & 0,25 \\
\hline 118. Interpublic Group Of Companies, Inc. & 0,25 & 172. BbyT Corp & 0,25 \\
\hline 119. Avery Dennison Corporation & 0,25 & 173. Centerpoint Energy Inc & 0,25 \\
\hline 120. Nucor Corp & 0,25 & 174. Mcdonalds Corp & 0,25 \\
\hline 121. Wyeth & 0,25 & 175. Lsi Logic Corp & 0,25 \\
\hline 122. Black y Decker Corp & 0,25 & 176. Sherwin Williams Co & 0,25 \\
\hline 123. Emc Corp & 0,25 & 177. American Electric Power Co Inc & 0,25 \\
\hline 124. Johnson Controls Inc & 0,25 & 178. Hilton Hotels Corp & 0,25 \\
\hline
\end{tabular}




\begin{tabular}{|c|c|c|c|}
\hline Razón social & $\mathbf{R}^{2}$ & Razón social & $\mathrm{R}^{2}$ \\
\hline 179. First Horizon National Corp & 0,25 & 233. King Pharmaceuticals Inc & 0,16 \\
\hline 180. Walt Disney Co/ & 0,25 & 234. J C Penney Co Inc & 0,16 \\
\hline 181. Bank Of America Corp /De/ & 0,25 & 235. Wal Mart Stores Inc & 0,16 \\
\hline 182. Marshall y Ilsley Corp/Wi/ & 0,25 & 236. News Corp & 0,16 \\
\hline 183. Alcoa Inc & 0,25 & 237. Mattel Inc /De/ & 0,16 \\
\hline 184. St Jude Medical Inc & 0,25 & 238. First Data Corp & 0,16 \\
\hline 185. Dillards Inc & 0,25 & 239. Sovereign Bancorp Inc & 0,16 \\
\hline 186. Sealed Air Corp/De & 0,25 & 240. Anadarko Petroleum Corp & 0,16 \\
\hline 187. Boeing Co & 0,25 & 241. Gap Inc & 0,16 \\
\hline 188. Southern Co & 0,25 & 242. Centurytel Inc & 0,16 \\
\hline 189. Ncr Corp & 0,25 & 243. Dollar General Corp & 0,16 \\
\hline 190. Watson Pharmaceuticals Inc & 0,25 & 244. Yum Brands Inc & 0,16 \\
\hline 191. Ca, Inc. & 0,25 & 245. Lee Sara Corp & 0,16 \\
\hline 192. Pinnacle West Capital Corp & 0,25 & 246. Equifax Inc & 0,16 \\
\hline 193. Xcel Energy Inc & 0,25 & 247. Countrywide Financial Corp & 0,16 \\
\hline 194. Sabre Holdings Corp & 0,25 & 248. Harman International Industries Inc /De/ & 0,16 \\
\hline 195. International Flavors y Fragrances Inc & 0,25 & 249. Estee Lauder Companies Inc & 0,16 \\
\hline 196. Commerce Bancorp Inc /Nj/ & 0,25 & 250. Baker Hughes Inc & 0,16 \\
\hline 197. Mcgraw-Hill Companies Inc & 0,25 & 251. Tjx Companies Inc /De/ & 0,16 \\
\hline 198. Convergys Corp & 0,25 & 252. Lockheed Martin Corp & 0,16 \\
\hline 199. Synovus Financial Corp & 0,25 & 253. Constellation Energy Group Inc & 0,16 \\
\hline 200. Washington Mutual, Inc & 0,25 & 254. Dominion Resources Inc $/ \mathrm{Na} /$ & 0,16 \\
\hline 201. Occidental Petroleum Corp /De/ & 0,25 & 255. Marathon Oil Corp & 0,16 \\
\hline 202. Valero Energy Corp/Tx & 0,25 & 256. Conocophillips & 0,16 \\
\hline 203. Motorola Inc & 0,25 & 257. Chesapeake Energy Corp & 0,16 \\
\hline 204. Southwest Airlines Co & 0,25 & 258. Unisys Corp & 0,16 \\
\hline 205. National Oilwell Varco Inc & 0,25 & 259. L 3 Communications Holdings Inc & 0,16 \\
\hline 206. Analog Devices Inc & 0,25 & 260. Transocean Inc & 0,16 \\
\hline 207. AtyT Inc. & 0,25 & 261. Pitney Bowes Inc /De/ & 0,16 \\
\hline 208. Reynolds American Inc & 0,25 & 262. Ecolab Inc & 0,16 \\
\hline 209. Noble Corp & 0,25 & 263. Corning Inc /Ny & 0,16 \\
\hline 210. Wendys International Inc & 0,25 & 264. Navistar International Corp & 0,16 \\
\hline 211. Weatherford International Ltd & 0,25 & 265. Edison International & 0,16 \\
\hline 212. Sunoco Inc & 0,25 & 266. Chicago Mercantile Exchange Holdings Inc & 0,16 \\
\hline 213. Ambac Financial Group Inc & 0,16 & 267. Progressive Corp/Oh/ & 0,16 \\
\hline 214. National City Corp & 0,16 & 268. Pnc Financial Services Group Inc & 0,16 \\
\hline 215. Cit Group Inc & 0,16 & 269. Kimberly Clark Corp & 0,16 \\
\hline 216. Aflac Inc & 0,16 & 270. Merck y Co Inc & 0,16 \\
\hline 217. Freeport Mcmoran Copper y Gold Inc & 0,16 & 271. Prudential Financial Inc & 0,16 \\
\hline 218. Xto Energy Inc & 0,16 & 272. Us Bancorp \De\ & 0,16 \\
\hline 219. Jabil Circuit Inc & 0,16 & 273. Ameren Corp & 0,16 \\
\hline 220. Mckesson Corp & 0,16 & 274. Chubb Corp & 0,16 \\
\hline 221. Affiliated Computer Services Inc & 0,16 & 275. Genworth Financial Inc & 0,16 \\
\hline 222. Safeco Corp & 0,16 & 276. Schlumberger Ltd /Nv/ & 0,16 \\
\hline 223. Omnicom Group Inc & 0,16 & 277. Stryker Corp & 0,16 \\
\hline 224. Keycorp /New/ & 0,16 & 278. Applera Corp & 0,16 \\
\hline 225. Ashland Inc & 0,16 & 279. Txu Corp /Tx/ & 0,16 \\
\hline 226. St Paul Travelers Companies Inc & 0,16 & 280. Consol Energy Inc & 0,16 \\
\hline 227. Northrop Grumman Corp /De/ & 0,16 & 281. V F Corp & 0,16 \\
\hline 228. Hasbro Inc & 0,16 & 282. Lexmark International Inc /Ky/ & 0,16 \\
\hline 229. Peoples Energy Corp & 0,16 & 283. Coca Cola Enterprises Inc & 0,16 \\
\hline 230. Lilly Eli y Co & 0,16 & 284. Exxon Mobil Corp & 0,16 \\
\hline 231. Goodyear Tire y Rubber Co /Oh/ & 0,16 & 285. Dte Energy Co & 0,16 \\
\hline 232. XI Capital Ltd & 0,16 & 286. Barr Pharmaceuticals Inc & 0,16 \\
\hline
\end{tabular}




\begin{tabular}{|c|c|c|c|}
\hline Razón social & $\mathrm{R}^{2}$ & Razón social & $\mathbf{R}^{2}$ \\
\hline 287. Avaya Inc & 0,16 & 341. Office Depot Inc & 0,09 \\
\hline 288. Micron Technology Inc & 0,16 & 342. Kinder Morgan Inc & 0,09 \\
\hline 289. Bard C R Inc /Nj/ & 0,16 & 343. Ford Motor Co & 0,09 \\
\hline 290. Viacom Inc. & 0,16 & 344. Allergan Inc & 0,09 \\
\hline 291. El Paso Corp/De & 0,16 & 345. Perkinelmer Inc & 0,09 \\
\hline 292. Altria Group, Inc. & 0,16 & 346. Autozone Inc & $0,0 \varsigma$ \\
\hline 293. Campbell Soup Co & 0,16 & 347. Kellogg Co & 0,09 \\
\hline 294. Limited Brands Inc & 0,16 & 348. Scripps E W Co /De & 0,09 \\
\hline 295. Firstenergy Corp & 0,16 & 349. Forest Laboratories Inc & 0,09 \\
\hline 296. Boston Scientific Corp & 0,16 & 350. General Mills Inc & 0,09 \\
\hline 297. Abbott Laboratories & 0,16 & 351. Dow Jones y Co Inc & 0,09 \\
\hline 298. Starwood Hotel y Resorts Worldwide Inc & 0,16 & 352. Sprint Nextel Corp & $0,0 \mathrm{~s}$ \\
\hline 299. Unumprovident Corp & 0,16 & 353. Alltel Corp & 0,09 \\
\hline 300. Entergy Corp /De/ & 0,16 & 354. Dynegy Inc /II/ & 0,09 \\
\hline 301. Halliburton $\mathrm{Co}_{0}$ & 0,16 & 355. Hershey Co & 0,09 \\
\hline 302. Wells Fargo y $\mathrm{Co} / \mathrm{Mn}$ & 0,16 & 356. General Motors Corp & 0,09 \\
\hline 303. Windstream Corp & 0,16 & 357. Hospira Inc & 0,09 \\
\hline 304. Comerica Inc /New/ & 0,16 & 358. Newmont Mining Corp /De/ & 0,04 \\
\hline 305. Chevron Corp & 0,16 & 359. Fpl Group Inc & 0,04 \\
\hline 306. Wrigley Wm Jr Co & 0,16 & 360. Harrahs Entertainment Inc & 0,04 \\
\hline 307. Safeway Inc & 0,16 & 361. Public Service Enterprise Group Inc & 0,04 \\
\hline 308. Brown Forman Corp & 0,16 & 362. Qwest Communications International Inc & 0,04 \\
\hline 309. Aon Corp & 0,16 & 363. Hess Corp & 0,04 \\
\hline 310. Ppl Corp & 0,16 & 364. New York Times Co & 0,04 \\
\hline 311. Legg Mason Inc & 0,09 & 365. Capital One Financial Corp & 0,04 \\
\hline 312. Mccormick y Co Inc & 0,09 & 366. Ust Inc & 0,04 \\
\hline 313. Marsh y Mclennan Companies, Inc. & 0,09 & 367. Radioshack Corp & 0,04 \\
\hline 314. General Dynamics Corp & 0,09 & 368. Laboratory Corp Of America Holdings & 0,04 \\
\hline 315. Automatic Data Processing Inc & 0,09 & 369. Meredith Corp & 0,04 \\
\hline 316. Consolidated Edison Inc & 0,09 & 370. Murphy Oil Corp /De & 0,04 \\
\hline 317. Bristol Myers Squibb Co & 0,09 & 371. Phelps Dodge Corp & 0,04 \\
\hline 318. Cms Energy Corp & 0,09 & 372. Jones Apparel Group Inc & 0,04 \\
\hline 319. Bmc Software Inc & 0,09 & 373. SIm Corp & 0,04 \\
\hline 320. United Parcel Service Inc & 0,09 & 374. Computer Sciences Corp & 0,04 \\
\hline 321. Nisource Inc/De & 0,09 & 375. Pepsi Bottling Group Inc & 0,04 \\
\hline 322. Exelon Corp & 0,09 & 376. Medtronic Inc & 0,04 \\
\hline 323. Coca Cola Co & 0,09 & 377. Avon Products Inc & 0,04 \\
\hline 324. Manor Care Inc & 0,09 & 378. Univision Communications Inc & 0,04 \\
\hline 325. Kohls Corporation & 0,09 & 379. Keyspan Corp & 0,04 \\
\hline 326. Smith International Inc & 0,09 & 380. Tenet Healthcare Corp & 0,04 \\
\hline 327. Cardinal Health Inc & 0,09 & 381. Tyson Foods Inc & 0,04 \\
\hline 328. Suntrust Banks Inc & 0,09 & 382. RR Donnelley y Sons Co & 0,04 \\
\hline 329. Archer Daniels Midland Co & 0,09 & 383. Zimmer Holdings Inc & 0,04 \\
\hline 330. Regions Financial Corp & 0,09 & 384. Dean Foods Co & 0,01 \\
\hline 331. Clear Channel Communications Inc & 0,09 & 385. Nike Inc & 0,01 \\
\hline 332. Anheuser-Busch Companies, Inc. & 0,09 & 386. Medco Health Solutions Inc & 0,01 \\
\hline 333. Time Warner Inc & 0,09 & 387. Pepsico Inc & 0,01 \\
\hline 334. HyR Block Inc & 0,09 & 388. Cvs Corp & 0,01 \\
\hline 335. Advanced Micro Devices Inc & 0,09 & 389. Walgreen Co & 0,01 \\
\hline 336. Equity Office Properties Trust & 0,09 & 390. Johnson y Johnson & 0,01 \\
\hline 337. Kroger Co & 0,09 & 391. Aes Corp & 0,01 \\
\hline 338. Ims Health Inc & 0,09 & 392. Conagra Foods Inc /De/ & 0,01 \\
\hline 339. Gannett Co Inc /De/ & 0,09 & 393. Sysco Corp & 0,01 \\
\hline 340. Baxter International Inc & 0,09 & 394. Quest Diagnostics Inc & 0,01 \\
\hline
\end{tabular}




\begin{tabular}{lc}
\hline \multicolumn{1}{c}{ Razón social } & $\mathbf{R}^{2}$ \\
\hline 395. Procter y Gamble Co & 0,01 \\
396. Bausch y Lomb Inc & 0,01 \\
397. Molson Coors Brewing Co & 0,01 \\
398. Coventry Health Care Inc & 0,01 \\
399. Health Management Associates Inc & 0,01 \\
400. Supervalu Inc & 0,01 \\
401. Aetna Inc /Pa/ & 0,01 \\
402. Constellation Brands, Inc. & 0 \\
403. Officemax Inc & 0 \\
404. Unitedhealth Group Inc & 0 \\
405. Humana Inc & 0 \\
406. Caremark Rx Inc & 0 \\
407. Tribune Co & 0 \\
408. Colgate Palmolive Co & 0 \\
409. Cigna Corp & 0 \\
410. Wellpoint Inc & 0 \\
411. Embarq Corp & 0 \\
412. Realogy Corp & 0 \\
413. Western Union Co & 0 \\
414. Wyndham Worldwide Corp & 0 \\
\hline
\end{tabular}

Fuente: Economática, 9 de febrero de 2007. un valor de corte del 20\% para el coeficiente de determinación.

En conclusión, la estrategia seleccionada es utilizar un $\mathrm{R}^{2}$ de 0,20 computado con datos semanales durante el plazo de un año.

\section{Empresas seleccionadas}

Utilizando el corte de $20 \%$ para los $\mathrm{R}^{2}$ con data semanal en el plazo de un año serían 81 las empresas latinoamericanas con acciones emitidas en sus bolsas locales susceptibles de utilizar el CAPM con un mínimo de confiabilidad. En el cuadro 8 se presentan los $\mathrm{R}^{2}$ para todas las empresas con acciones en sus bolsas locales que superaron los criterios anteriores.

Cuadro 8. Empresas de América Latina con acciones líquidas en su mercado local y cotizaciones con $\mathrm{R}^{2}$ significativo respecto del S\&P 500

\begin{tabular}{|c|c|c|c|c|c|}
\hline Razón social & Sector económico & País sede & $\begin{array}{l}\text { Presencia } \\
\quad(\%)\end{array}$ & $\begin{array}{l}\text { Volumen } \\
\text { transado } \\
\text { (dólares) }\end{array}$ & $\begin{array}{c}\mathrm{R}^{2} \text { respecto } \\
\text { del SyP } 500 \\
\text { (cotizado en } \\
\text { dólares) }\end{array}$ \\
\hline 1. Enersis S. A. & Energía eléctrica & Chile & 100 & 6309294 & 0,5929 \\
\hline 2. Banco Bradesco S/A & Finanzas y Seguros & Brasil & 100 & 38665501 & 0,5776 \\
\hline $\begin{array}{l}\text { 3. Banco Itau Holding } \\
\text { Financeira S/A }\end{array}$ & Finanzas y Seguros & Brasil & 100 & 27473530 & 0,5776 \\
\hline $\begin{array}{l}\text { 4. Desarrolladora } \\
\text { Homex S. A. de C. V. }\end{array}$ & Construcción & México & 100 & 6972275 & 0,5476 \\
\hline $\begin{array}{l}\text { 5. Unibanco (Uniao de Bancos } \\
\text { Brasileiros S/A) }\end{array}$ & Finanzas y Seguros & Brasil & 100 & 15986789 & 0,5476 \\
\hline $\begin{array}{l}\text { 6. Tele Norte Leste } \\
\text { Participações S/A }\end{array}$ & Telecomunicación & Brasil & 100 & 25721510 & 0,5329 \\
\hline $\begin{array}{l}\text { 7. Empresa Nacional de } \\
\text { Electricidad S. A. }\end{array}$ & Energía eléctrica & Chile & 100 & 3180513 & 0,5041 \\
\hline 8. Tim Participacoes S/A & Telecomunicación & Brasil & 100 & 8328673 & 0,5041 \\
\hline $\begin{array}{l}\text { 9. Itausa (Investimentos } \\
\text { Itau S/A) }\end{array}$ & Otros & Brasil & 100 & 23456472 & 0,4761 \\
\hline 10. Cemex, S. A. de C.V. & Minerales no metálicos & México & 100 & 43481958 & 0,4624 \\
\hline $\begin{array}{l}\text { 11. Companhia de Bebidas } \\
\text { Das Americas (Ambev) }\end{array}$ & Alimentos y Bebidas & Brasil & 100 & 20840732 & 0,4624 \\
\hline
\end{tabular}




\section{Razón social}

Sector económico País sede

Alimentos y Bebidas
Telecomunicación
Transporte y Servicios
Papel y Celulosa
Otros
Comercio
Papel y Celulosa
Papel y Celulosa
Comercio

Telecomunicación

Telecomunicación

Telecomunicación

Telecomunicación

Papel y Celulosa

Transporte y Servicios

Finanzas y Seguros

Papel y Celulosa Transporte y Servicios

Finanzas y Seguros Comercio Minería

Energía eléctrica Comercio

Telecomunicación

Finanzas y Seguros

Petróleo y Gas

Siderurgia y Metalurgia

$$
\begin{gathered}
\text { Comercio } \\
\text { Otros } \\
\text { Minería }
\end{gathered}
$$

Alimentos y Bebidas Otros

Siderurgia y Metalurgia

Siderurgia y Metalurgia

Finanzas y Seguros

Petróleo y Gas
México

Brasil

Brasil

México

México

Chile

Brasil

Brasil

Brasil

México

Brasil

Brasil

Chile

Brasil

Chile

México

Brasil

Brasil

Chile

Chile

Brasil

Colombia

Brasil

Brasil

Chile

Brasil

Brasil

Chile

Argentina

México

México

Brasil

Brasil

Brasil

Colombia

Brasil

\section{Presencia}

(\%)
$\mathrm{R}^{2}$ respecto

del S\&P 500

(cotizado en dólares)

0,4624

0,4624

0,4225

0,4225

0,3969

0,3969

0,3969

0,3844

0,3844

0,3721

0,3721

0,3721

0,3600

0543697

0,3600

5990128

0,3600

100

6076116

0,3481

\section{0}

100

100

100

100

100

100

100

13648883

9264557

3331593

9644716

150180994

3318465

22435931

1607180

0,3481

0,3481

0,3364

0,3364

0,3364

0,3364

0,3364

0,3364

$100 \quad 633341$

0,3249

2132619

0,3249

$100 \quad 33202742$

0,3249

100

3207187

0,3249

1716474

0,3249

29197726

0,3249

7190253

0,3249

26184378

0,3136

7717144

0,3136

38857661

0,3136 Minas Gerais SA

46. Cia. Suramericana de Inversiones

47. Petroleo Brasileiro S/A (Petrobras) 


\begin{tabular}{|c|c|c|c|c|c|c|}
\hline & Razón social & Sector económico & País sede & $\begin{array}{l}\text { Presencia } \\
(\%)\end{array}$ & $\begin{array}{l}\text { Volumen } \\
\text { transado } \\
\text { (dólares) }\end{array}$ & $\begin{array}{c}\mathrm{R}^{2} \text { respecto } \\
\text { del S\&P } 500 \\
\text { (cotizado en } \\
\text { dólares) }\end{array}$ \\
\hline 48. & Tractebel Energia SA & Energía eléctrica & Brasil & 100 & 3657187 & 0,3025 \\
\hline 49. & $\begin{array}{l}\text { Cia. Saneamento Basico } \\
\text { Estado Sao Paulo }\end{array}$ & Otros & Brasil & 100 & 2150095 & 0,2916 \\
\hline 50. & Gerdau SA & Siderurgia y Metalurgia & Brasil & 100 & 26101080 & 0,2916 \\
\hline 51. & Brasil Telecom SA & Telecomunicación & Brasil & 100 & 13901411 & 0,2809 \\
\hline 52. & Consorcio ARA, S. A. de C. V. & Construcción & México & 100 & 4931838 & 0,2809 \\
\hline 53. & $\begin{array}{l}\text { Copesul (Companhia } \\
\text { Petroquimica do Sul) }\end{array}$ & Química & Brasil & 100 & 2340576 & 0,2809 \\
\hline 54. & $\begin{array}{l}\text { Net Servicos de } \\
\text { Comunicacao SA }\end{array}$ & Otros & Brasil & 100 & 30473820 & 0,2809 \\
\hline 55. & Inversiones Argos S. A. & Finanzas y Seguros & Colombia & 100 & 1574458 & 0,2704 \\
\hline 56. & Lojas Americanas SA & Comercio & Brasil & 100 & 2181822 & 0,2704 \\
\hline 57. & $\begin{array}{l}\text { Companhia Energetica de } \\
\text { Minas Gerais }\end{array}$ & Energía eléctrica & Brasil & 100 & 12444141 & 0,2601 \\
\hline 58. & Diagnosticos da America SA & Otros & Brasil & 100 & 2877559 & 0,2601 \\
\hline 59. & Obrascon Huarte Lain Brasil SA & Transporte y Servicios & Brasil & 100 & 718585 & 0,2601 \\
\hline 60. & $\begin{array}{l}\text { Sociedad Química y Minera } \\
\text { de Chile S. A. }\end{array}$ & Minería & Chile & 100 & 636916 & 0,2601 \\
\hline 61. & CPFL Energia SA & Energía eléctrica & Brasil & 100 & 8571291 & 0,2500 \\
\hline 62. & Embotelladora Andina S. A. & Alimentos y Bebidas & Chile & 100 & 668691 & 0,2500 \\
\hline 63. & $\begin{array}{l}\text { Embrear (Emp Brasileira } \\
\text { Aeronautica SA) }\end{array}$ & Vehículos y Piezas & Brasil & 100 & 12782519 & 0,2500 \\
\hline 64. & Gruma S. A. de C. V. & Alimentos y Bebidas & México & 100 & 8448891 & 0,2500 \\
\hline 65. & $\begin{array}{l}\text { Grupo Aeroportuario, } \\
\text { S.A. de C.V. }\end{array}$ & Transporte y Servicios & México & 100 & 1599487 & 0,2500 \\
\hline 66. & Grupo Carso, S.A. de C.V. & Otros & México & 100 & 2616097 & 0,2500 \\
\hline 67. & Telecom Argentina S. A. & Telecomunicación & Argentina & 100 & 2657257 & 0,2500 \\
\hline 68. & Eternit SA & Minerales no metálicos & Brasil & 100 & 1647539 & 0,2401 \\
\hline 69. & Cementos Argos S. A. & Minerales no metálicos & Colombia & 100 & 2328403 & 0,2304 \\
\hline 70. & Cía. Colombiana de Inversiones & Finanzas y Seguros & Colombia & 100 & 969551 & 0,2304 \\
\hline 71. & $\begin{array}{l}\text { Cia. Paranaense de Energia } \\
\text { (Copel) }\end{array}$ & Energía eléctrica & Brasil & 100 & 8489250 & 0,2304 \\
\hline 72. & EDP (Energias do Brasil SA) & Energía eléctrica & Brasil & 100 & 2930754 & 0,2304 \\
\hline 73. & Braskem SA & Química & Brasil & 100 & 8706350 & 0,2209 \\
\hline 74. & Empresas CMPC S. A. & Papel y Celulosa & Chile & 100 & 2599753 & 0,2209 \\
\hline 75. & $\begin{array}{l}\text { Petrobrás Energía } \\
\text { Participaciones S. A. }\end{array}$ & Otros & Argentina & 100 & 1503679 & 0,2209 \\
\hline 76. & $\begin{array}{l}\text { Urbi Desarrollos Urbanos, } \\
\text { S. A. de C.V. }\end{array}$ & Construcción & México & 100 & 5513451 & 0,2209 \\
\hline 77. & $\begin{array}{l}\text { Corporación Financiera } \\
\text { Colombiana S. A. }\end{array}$ & Finanzas y Seguros & Colombia & 100 & 12498973 & 0,2116 \\
\hline 78. & Sadia SA & Alimentos y Bebidas & Brasil & 100 & 4065734 & 0,2116 \\
\hline 79. & Localiza Rent a Car SA & Transporte y Servicios & Brasil & 100 & 16048650 & 0,2025 \\
\hline 80. & Tenaris S. A. & Siderurgia y Metalurgia & Argentina & 100 & 1240259 & 0,2025 \\
\hline 81. & $\begin{array}{l}\text { Unipar (Uniao Inds. } \\
\text { Petroquimicas S/A) }\end{array}$ & Química & Brasil & 100 & 876661 & 0,2025 \\
\hline
\end{tabular}

Fuente: Economática, 9 de febrero de 2007. 


\section{Cuadro 9. Empresas de América Latina con ADR líquidos y} cotizaciones con $\mathrm{R}^{2}$ significativo respecto del S\&P 500

\begin{tabular}{|c|c|c|c|c|c|}
\hline Razón social & Sector económico & País sede & $\begin{array}{l}\text { Presencia } \\
(\%)\end{array}$ & $\begin{array}{l}\text { Volumen } \\
\text { transado } \\
\text { (dólares) }\end{array}$ & $\begin{array}{c}\mathrm{R}^{2} \text { respecto } \\
\text { del S\&P } 500 \\
\text { (cotizado en } \\
\text { dólares) }\end{array}$ \\
\hline 1. Enersis S. A. & Energía eléctrica & Chile & 100 & 7805966 & 0,6400 \\
\hline 2. Banco Bradesco SA & Finanzas y Seguros & Brasil & 100 & 79292565 & 0,6084 \\
\hline $\begin{array}{l}\text { 3. Banco Itau Holding } \\
\text { Financeira SA }\end{array}$ & Finanzas y Seguros & Brasil & 100 & 50745123 & 0,5776 \\
\hline $\begin{array}{l}\text { 4. Tele Norte Leste } \\
\text { Participações S/A }\end{array}$ & Telecomunicaciones & Brasil & 100 & 24940475 & 0,5776 \\
\hline $\begin{array}{l}\text { 5. Unibanco (Uniao de Bancos } \\
\text { Brasileiros SA) }\end{array}$ & Finanzas y Seguros & Brasil & 100 & 81297882 & 0,5776 \\
\hline $\begin{array}{l}\text { 6. Desarrolladora Homex, } \\
\text { S.A. de C.V. }\end{array}$ & Construcción & México & 100 & 27062442 & 0,5329 \\
\hline 7. Cemex, S.A. de C.V. & Minerales no metálicos & México & 100 & 79405776 & 0,5184 \\
\hline $\begin{array}{l}\text { 8. Companhia de Bebidas } \\
\text { Das Americas (Ambev) }\end{array}$ & Alimentos y Bebidas & Brasil & 100 & 27787152 & 0,5041 \\
\hline 9. Tim Participacoes SA & Telecomunicación & Brasil & 100 & 10376493 & 0,4900 \\
\hline $\begin{array}{l}\text { 10. Fomento Económico } \\
\text { Mexicano, S. A. de C.V. }\end{array}$ & Alimentos y Bebidas & México & 100 & 40063920 & 0,4761 \\
\hline $\begin{array}{l}\text { 11. Empresa Nacional de } \\
\text { Electricidad S. A. }\end{array}$ & Energía eléctrica & Chile & 100 & 790725 & 0,4624 \\
\hline $\begin{array}{l}\text { 12. Gol Linhas Aéreas } \\
\text { Inteligentes S/A }\end{array}$ & Transporte y Servicios & Brasil & 100 & 18662992 & 0,4225 \\
\hline 13. Grupo Televisa, S. A. de C. V. & Otros & México & 100 & 47807421 & 0,4225 \\
\hline 14. América Móvil, S. A. de C.V. & Telecomunicación & México & 100 & 326177280 & 0,4096 \\
\hline 15. Aracruz Celulose SA & Papel y Celulosa & Brasil & 100 & 19187014 & 0,4096 \\
\hline 16. Brasil Telecom Participacoes SA & A Telecomunicación & Brasil & 100 & 30994637 & 0,3969 \\
\hline 17. BBVA Banco Francés S. A. & Finanzas y Seguros & Argentina & 100 & 1104985 & 0,3844 \\
\hline 18. Cia. Vale do Rio Doce & Minería & Brasil & 100 & 185563425 & 0,3844 \\
\hline 19. Banco Colombia & Finanzas y Seguros & Colombia & 100 & 8171628 & 0,3721 \\
\hline 20. Vivo Participacoes S/A & Telecomunicación & Brasil & 100 & 10640505 & 0,3721 \\
\hline 21. Lan Airlines S. A. & Transporte y Servicios & Chile & 100 & 8028288 & 0,3600 \\
\hline $\begin{array}{l}\text { 22. Companhia Brasileira de } \\
\text { Distribuicao }\end{array}$ & Comercio & Brasil & 100 & 5643686 & 0,3600 \\
\hline 23. Banco Santander-Chile & Finanzas y Seguros & Chile & 100 & 7285026 & 0,3481 \\
\hline 24. Telecom Argentina S. A. & Telecomunicación & Argentina & 100 & 3968002 & 0,3364 \\
\hline 25. Votorantim Celulose e Papel SA & Papel y Celulosa & Brasil & 100 & 7349390 & 0,3136 \\
\hline $\begin{array}{l}\text { 26. Companhia Siderurgica } \\
\text { Nacional }\end{array}$ & Siderurgia y Metalurgia & Brasil & 100 & 35345667 & 0,3025 \\
\hline $\begin{array}{l}\text { 27. Sociedad Química y Minera } \\
\text { de Chile S. A. }\end{array}$ & Minería & Chile & 100 & 3885120 & 0,3025 \\
\hline 28. Brasil Telecom SA & Telecomunicación & Brasil & 100 & 1827000 & 0,2916 \\
\hline $\begin{array}{l}\text { 29. Petroleo Brasileiro S/A } \\
\text { (Petrobras) }\end{array}$ & Petróleo y Gas & Brasil & 100 & 190480695 & 0,2916 \\
\hline $\begin{array}{l}\text { 30. Telecomunicacoes de Sao } \\
\text { Paulo S/A (Telesp) }\end{array}$ & Telecomunicación & Brasil & 100 & 1783733 & 0,2916 \\
\hline 31. Gerdau SA & Siderurgia y Metalurgia & Brasil & 100 & 24366168 & 0,2809 \\
\hline $\begin{array}{l}\text { 32. Cia. Saneamento Basico } \\
\text { Estado Sao Paulo }\end{array}$ & Otros & Brasil & 100 & 8043350 & 0,2809 \\
\hline 33. Ternium & Siderurgia y Metalurgia & Argentina & 100 & 3906495 & 0,2704 \\
\hline
\end{tabular}




\begin{tabular}{|c|c|c|c|c|c|c|}
\hline & Razón social & Sector económico & País sede & $\begin{array}{l}\text { Presencia } \\
(\%)\end{array}$ & $\begin{array}{l}\text { Volumen } \\
\text { transado } \\
\text { (dólares) }\end{array}$ & $\begin{array}{c}\mathrm{R}^{2} \text { respecto } \\
\text { del } \mathrm{S} \& \mathrm{P} 500 \\
\text { (cotizado en } \\
\text { dólares) }\end{array}$ \\
\hline 34. & $\begin{array}{l}\text { Companhia Energetica de } \\
\text { Minas Gerais }\end{array}$ & Energía eléctrica & Brasil & 100 & 17212824 & 0,2601 \\
\hline 35. & $\begin{array}{l}\text { Cia. Paranaense de Energia } \\
\text { (Copel) }\end{array}$ & Energía eléctrica & Brasil & 100 & 3673004 & 0,2601 \\
\hline 36. & $\begin{array}{l}\text { Petrobrás Energia } \\
\text { Participaciones S. A. }\end{array}$ & Otros & Argentina & 100 & 1493865 & 0,2601 \\
\hline & $\begin{array}{l}\text { Cía. de Telecomunicaciones } \\
\text { de Chile S. A. }\end{array}$ & Telecomunicación & Chile & 100 & 4797045 & 0,2401 \\
\hline 38. & Tenaris S. A. & Siderurgia y Metalurgia & Argentina & 100 & 63576071 & 0,2401 \\
\hline 39. & Braskem SA & Química & Brasil & 100 & 3852206 & 0,2304 \\
\hline 40. & Coca Cola Femsa, S. A. de C.V. & Alimentos y Bebidas & México & 100 & 7241488 & 0,2304 \\
\hline 41. & Masisa S. A. & Agro y Pesca & Chile & 100 & 6072192 & 0,2304 \\
\hline 42. & Sadia SA & Alimentos y Bebidas & Brasil & 100 & 4743530 & 0,2304 \\
\hline 43. & CPFL Energia SA & Energía eléctrica & Brasil & 100 & 3187032 & 0,2209 \\
\hline & $\begin{array}{l}\text { Teléfonos de México, } \\
\text { S.A. de C.V. }\end{array}$ & Telecomunicación & México & 100 & 24871410 & 0,2025 \\
\hline
\end{tabular}

Fuente: Economática, 9 de febrero de 2007.

Igualmente, serían 44 las empresas latinoamericanas con ADR emitidos en la Bolsa de Nueva York para las que es aceptable utilizar el CAPM con un mínimo de confiabilidad. El cuadro 9 muestra los $\mathrm{R}^{2}$ para todas las empresas con ADR que han pasado los criterios impuestos.
Por tanto, son 81 las empresas que se cotizan en sus bolsas locales y 44 las empresas que han emitido ADR en la Bolsa de Nueva York que pasan todos los filtros (ver cuadro 10). Puesto que algunas de las empresas aparecen en ambas listas, se puede concluir que el número total de

\section{Cuadro 10. Empresas de América Latina que pueden calcular beta directamente}

\begin{tabular}{lcc}
\hline \multicolumn{1}{c}{ Razón social } & Sector económico & País sede \\
\hline 1. BBVA Banco Francés S. A. & Finanzas y Seguros & Argentina \\
2. Grupo Financiero Galicia S. A. & Otros & Argentina \\
3. Petrobrás Energia Participaciones S. A. & Otros & Argentina \\
4. Telecom Argentina S. A. & Telecomunicación & Argentina \\
5. Tenaris S. A. & Siderurgia y Metalurgia & Argentina \\
6. Ternium & Siderurgia y Metalurgia & Argentina \\
7. Aracruz Celulose SA & Papel y Celulosa & Brasil \\
8. Banco Bradesco SA & Finanzas y Seguros & Brasil \\
9. Banco Itau Holding Financeira SA & Finanzas y Seguros & Brasil \\
10. Bradespar SA & Otros & Brasil \\
11. Brasil Telecom Participacoes SA & Telecomunicación & Brasil \\
12. Brasil Telecom SA & Telecomunicación & Brasil \\
13. Braskem SA & Química & Brasil \\
14. Cia. Saneamento Basico Estado Sao Paulo & Otros & Brasil \\
15. Cia. Vale do Rio Doce & Minería & Brasil \\
16. Cia. Brasileira de Petroleo Ipiranga & Petróleo y Gas & Brasil \\
17. Cia. Paranaense de Energia (Copel) & Energía eléctrica & Brasil \\
18. Companhia Brasileira de Distribuicao & Comercio & Brasil \\
\hline
\end{tabular}




\begin{tabular}{|c|c|c|}
\hline Razón social & Sector económico & País sede \\
\hline 19. Companhia de Bebidas Das Americas (Ambev) & Alimentos y Bebidas & Brasil \\
\hline 20. Companhia Energetica de Minas Gerais & Energía eléctrica & Brasil \\
\hline 21. Companhia Siderurgica Nacional & Siderurgia y Metalurgia & Brasil \\
\hline 22. Copesul (Companhia Petroquimica do Sul) & Química & Brasil \\
\hline 23. CPFL Energia SA & Energía eléctrica & Brasil \\
\hline 24. Diagnosticos da America SA & Otros & Brasil \\
\hline 25. EDP (Energias do Brasil SA) & Energía eléctrica & Brasil \\
\hline 26. Embraer (Emp Brasileira Aeronautica SA) & Vehículos y Piezas & Brasil \\
\hline 27. Eternit SA & Minerales no metálicos & Brasil \\
\hline 28. Gerdau SA & Siderurgia y Metalurgia & Brasil \\
\hline 29. Gol Linhas Aéreas Inteligentes S/A & Transporte y Servicios & Brasil \\
\hline 30. Itausa (Investimentos Itau SA) & Otros & Brasil \\
\hline 31. Klabin SA & Papel y Celulosa & Brasil \\
\hline 32. Localiza Rent a Car SA & Transporte y Servicios & Brasil \\
\hline 33. Lojas Americanas SA & Comercio & Brasil \\
\hline 34. Lojas Renner SA & Comercio & Brasil \\
\hline 35. Metalurgica Gerdau SA & Siderurgia y Metalurgia & Brasil \\
\hline 36. Net Servicos de Comunicacao SA & Otros & Brasil \\
\hline 37. Obrascon Huarte Lain Brasil SA & Transporte y Servicios & Brasil \\
\hline 38. Petroleo Brasileiro S/A (Petrobras) & Petróleo y Gas & Brasil \\
\hline 39. Sadia SA. & Alimentos y Bebidas & Brasil \\
\hline 40. Suzano Papel e Celulose SA & Papel y Celulosa & Brasil \\
\hline 41. Tam SA & Transporte y Servicios & Brasil \\
\hline 42. Tele Norte Leste Participações S/A & Telecomunicación & Brasil \\
\hline 43. Telecomunicacoes de Sao Paulo S/A (Telesp) & Telecomunicación & Brasil \\
\hline 44. Telemar Norte Leste S/A & Telecomunicación & Brasil \\
\hline 45. Tim Participacoes SA & Telecomunicación & Brasil \\
\hline 46. Tractebel Energia SA & Energía eléctrica & Brasil \\
\hline 47. Unibanco (Uniao de Bancos Brasileiros SA) & Finanzas y Seguros & Brasil \\
\hline 48. Unipar (Uniao Inds. Petroquimicas $S / A$ ) & Química & Brasil \\
\hline 49. Usinas Siderurgicas de Minas Gerais SA & Siderurgia y Metalurgia & Brasil \\
\hline 50. Vivo Participacoes S/A & Telecomunicación & Brasil \\
\hline 51. Votorantim Celulose e Papel SA & Papel y Celulosa & Brasil \\
\hline 52. Banco Crédito e Inversiones & Finanzas y Seguros & Chile \\
\hline 53. Banco Santander-Chile & Finanzas y Seguros & Chile \\
\hline 54. Cencosud S. A. & Comercio & Chile \\
\hline 55. Cía. de Telecomunicaciones de Chile S. A. & Telecomunicación & Chile \\
\hline 56. Embotelladora Andina S. A. & Alimentos y Bebidas & Chile \\
\hline 57. Empresa Nacional de Electricidad S. A. & Energía eléctrica & Chile \\
\hline 58. Empresa Nacional de Telecomunicaciones S. A. & Telecomunicación & Chile \\
\hline 59. Empresas CMPC S. A. & Papel y Celulosa & Chile \\
\hline 60. Empresas La Polar S. A. & Comercio & Chile \\
\hline 61. Enersis S. A. & Energía eléctrica & Chile \\
\hline 62. Lan Airlines S. A. & Transporte y Servicios & Chile \\
\hline 63. Masisa S. A. & Agro y Pesca & Chile \\
\hline 64. Ripley Corp. S. A. & Comercio & Chile \\
\hline 65. Sociedad Química y Minera de Chile S. A. & Minería & Chile \\
\hline 66. Banco Colombia & Finanzas y Seguros & Colombia \\
\hline 67. Cementos Argos S. A. & Minerales no metálicos & Colombia \\
\hline 68. Cia. Colombiana de Inversiones & Finanzas y Seguros & Colombia \\
\hline 69. Cia. Suramericana de Inversiones & Finanzas y Seguros & Colombia \\
\hline 70. Corporacion Financiera Colombiana S. A. & Finanzas y Seguros & Colombia \\
\hline 71. I. S. A. - Previligiadas & Energía eléctrica & Colombia \\
\hline 72. Inversiones Argos S. A. & Finanzas y Seguros & Colombia \\
\hline
\end{tabular}




\begin{tabular}{lcc}
\hline \multicolumn{1}{c}{ Razón social } & Sector económico & País sede \\
\hline 73. América Móvil, S. A. de C.V. & Telecomunicación & México \\
74. Cemex, S. A. de C. V. & Minerales no metálicos & México \\
75. Coca Cola Femsa, S. A. de C.V. & Alimentos y Bebidas & México \\
76. Consorcio ARA, S. A. de C. V. & Construcción & México \\
77. Desarrolladora Homex, S. A. de C.V. & Construcción & México \\
78. Fomento Económico Mexicano, S. A. de C.V. & Alimentos y Bebidas & México \\
79. Gruma, S. A. de C. V. & Alimentos y Bebidas & México \\
80. Grupo Aeroportuario, S. A. de C.V. & Transporte y Servicios & México \\
81. Grupo Carso, S. A. de C.V. & Otros & México \\
82. Grupo Financiero Banorte, S. A. de C.V. & Finanzas y Seguros & México \\
83. Grupo México, S. A. de C.V. & Minería & México \\
84. Grupo Modelo, S. A. de C.V. & Alimentos y Bebidas & México \\
85. Grupo Televisa, S. A. de C.V. & Otros & México \\
86. Kimberly-Clark de México, S. A. de C.V. & Papel y Celulosa & México \\
87. Telefonos de México, S. A. de C.V. & Telecomunicación & México \\
88. Urbi Desarrollos Urbanos, S. A. de C.V. & Construcción & \\
\hline
\end{tabular}

empresas latinoamericanas que a la fecha de corte cumplen con todos los requisitos de liquidez y confiabilidad en el cómputo de sus betas es 88 .

\section{Conclusiones}

El cómputo de la tasa de descuento para empresas en países emergentes mediante la adición de una prima por riesgo-país a una tasa de descuento equivalente en un país desarrollado es una metodología débil. Resulta preferible utilizar el beta propio de cada empresa analizada puesto que solo así se preserva la consistencia con el CAPM al reflejarse en la tasa de descuento únicamente el componente sistemático del riesgopaís. Desafortunadamente, la estimación directa de beta es factible solo para aquellas empresas cuyas acciones se cotizan públicamente con suficiente liquidez.

En este trabajo se ha identificado este grupo privilegiado de empresas mediante la aplicación de los criterios de volumen y frecuencia de las cotizaciones de sus títulos, incluyendo tanto las acciones cotizadas en las bolsas nacionales como los ADR que se negocian en la Bolsa de Nueva York, y se ha encontrado que un total de 88 empresas de la región cumplen con los requisitos impuestos a la fecha de corte ( 9 de febrero de 2007). Es posible que los indicadores de liquidez sean inestables, por lo que el grupo de empresas calificadas puede cambiar a través del tiempo. Así, empresas no calificadas podrían en el futuro cumplir con los requisitos, y empresas que han pasado los filtros pueden dejar de hacerlo. Por esta razón es necesario actualizar el análisis con cierta frecuencia.

Es importante precisar que las tasas de descuento estimadas para las empresas seleccionadas serían las correctas para sus inversiones en un nivel corporativo, pero no para las inversiones que estas firmas realizasen en áreas geográficas específicas en las cuales entraría en juego el impacto del riesgo-país de las economías en cuestión. Queda pendiente la exploración de las posibilidades de estimación de estas tasas. También queda pendiente buscar una solución conceptualmente sólida para la estimación de las tasas de descuento corporativas de aquellas empresas que no pasan los criterios de liquidez. 


\section{Anexo 1 \\ Empresas emisoras de América Latina con liquidez en sus mercados locales}

\begin{tabular}{|c|c|c|c|c|c|c|}
\hline & Razón social & Sector económico & País sede & $\begin{array}{l}\text { Presencia } \\
\quad(\%)\end{array}$ & $\begin{array}{l}\text { Volumen } \\
\text { transado } \\
\text { (dólares) }\end{array}$ & $\begin{array}{c}\mathrm{R}^{2} \text { respecto } \\
\text { del S\&P } 500 \\
\text { (cotizado en } \\
\text { dólares) }\end{array}$ \\
\hline 1. & Pampa Holding S. A. & Alimentos y Bebidas & Argentina & 100 & 2102214 & 0,0256 \\
\hline 2. & Banco Macro Bansud S. A. & Finanzas y Seguros & Argentina & 100 & 1016885 & 0,1600 \\
\hline 3. & Grupo Financiero Galicia S. A. & Otros & Argentina & 100 & 1716474 & 0,3249 \\
\hline & $\begin{array}{l}\text { Petrobrás Energia } \\
\text { Participaciones S. A. }\end{array}$ & Otros & Argentina & 100 & 1503679 & 0,2209 \\
\hline 5. & $\begin{array}{l}\text { Siderar Sociedad Anónima } \\
\text { Industrial y Comercial }\end{array}$ & Siderurgia y Metalurgia & Argentina & 100 & 745549 & 0,1296 \\
\hline 6. & Tenaris S. A. & Siderurgia y Metalurgia & Argentina & 100 & 1240259 & 0,2025 \\
\hline 7. & Telecom Argentina S. A. & Telecomunicación & Argentina & 100 & 2657257 & 0,2500 \\
\hline & $\begin{array}{l}\text { Companhia de Bebidas } \\
\text { Das Americas (Ambev) }\end{array}$ & Alimentos y Bebidas & Brasil & 100 & 8831829 & 0,4761 \\
\hline 9. & Cosan SA Industria e Comercio & Alimentos y Bebidas & Brasil & 100 & 29913990 & 0,1764 \\
\hline 10. & $\begin{array}{l}\text { M Dias Branco S/A Industria } \\
\text { e Comercio de Alimentos }\end{array}$ & Alimentos y Bebidas & Brasil & 100 & 1193391 & - \\
\hline 11. & Perdigao SA & Alimentos y Bebidas & Brasil & 100 & 4304790 & 0,0529 \\
\hline 12. & Sadia SA & Alimentos y Bebidas & Brasil & 100 & 4065734 & 0,2116 \\
\hline 13. & $\begin{array}{l}\text { Companhia Brasileira de } \\
\text { Distribuicao }\end{array}$ & Comercio & Brasil & 100 & 6986884 & 0,3844 \\
\hline 14. & Lojas Americanas SA & Comercio & Brasil & 100 & 2181822 & 0,2704 \\
\hline 15. & Lojas Renner SA & Comercio & Brasil & 100 & 22435931 & 0,3364 \\
\hline 16. & Natura Cosmeticos S/A & Comercio & Brasil & 100 & 17157256 & 0,1681 \\
\hline 17. & Submarino S/A & Comercio & Brasil & 100 & 12587515 & 0,0900 \\
\hline & $\begin{array}{l}\text { Abyara Planejamento } \\
\text { Imobiliario SA }\end{array}$ & Construcción & Brasil & 100 & 4104953 & - \\
\hline 19. & Brazil Realty SA Emprs e Parts & Construcción & Brasil & 100 & 8853302 & 0,1369 \\
\hline 20. & Gafisa S/A & Construcción & Brasil & 100 & 5458686 & 0,1024 \\
\hline 21. & Klabin Segall S/A & Construcción & Brasil & 100 & 2177824 & - \\
\hline 22. & $\begin{array}{l}\text { LPS Brasil Consultoria de } \\
\text { Imoveis S/A }\end{array}$ & Construcción & Brasil & 100 & 2027385 & - \\
\hline 23. & Rossi Residencial S/A & Construcción & Brasil & 100 & 3728397 & 0,1024 \\
\hline 24. & Positivo Informatica S/A & Electroelectrónica & Brasil & 100 & 5353447 & - \\
\hline 25. & AES Tiete SA & Energía eléctrica & Brasil & 100 & 2780798 & 0,0529 \\
\hline 26. & Centrais Elet Brasileiras SA & Energía eléctrica & Brasil & 100 & 14445058 & 0,1600 \\
\hline 27. & $\begin{array}{l}\text { Centrais Eletricas de Santa } \\
\text { Catarina SA }\end{array}$ & Energía eléctrica & Brasil & 100 & 1788683 & 0,1600 \\
\hline 28. & $\begin{array}{l}\text { CESP (Companhia Energetica } \\
\text { de Sao Paulo) }\end{array}$ & Energía eléctrica & Brasil & 100 & 14565999 & - \\
\hline 29. & Cia. Energ Ceara (Coerce) & Energía eléctrica & Brasil & 100 & 745053 & 0,0081 \\
\hline 30. & $\begin{array}{l}\text { Cia. Paranaense de Energia } \\
\text { (Copel) }\end{array}$ & Energía eléctrica & Brasil & 100 & 8489250 & 0,2304 \\
\hline 31. & $\begin{array}{l}\text { Companhia Energetica de } \\
\text { Minas Gerais }\end{array}$ & Energía eléctrica & Brasil & 100 & 12444141 & 0,2601 \\
\hline 32. & CPFL Energia SA & Energía eléctrica & Brasil & 100 & 8571291 & 0,2500 \\
\hline & $\begin{array}{l}\text { CTEEP (Cia Transm Energia } \\
\text { Eletr. Paulista) }\end{array}$ & Energía eléctrica & Brasil & 100 & 11029192 & 0,0529 \\
\hline
\end{tabular}




\begin{tabular}{|c|c|c|c|c|c|c|}
\hline & Razón social & Sector económico & País sede & $\begin{array}{l}\text { Presencia } \\
(\%)\end{array}$ & $\begin{array}{l}\text { Volumen } \\
\text { transado } \\
\text { (dólares) }\end{array}$ & $\begin{array}{c}\mathrm{R}^{2} \text { respecto } \\
\text { del S\&P } 500 \\
\text { (cotizado en } \\
\text { dólares) }\end{array}$ \\
\hline 34. & EDP (Energias do Brasil SA) & Energía eléctrica & Brasil & 100 & 2930754 & 0,2304 \\
\hline 35. & $\begin{array}{l}\text { Eletropaulo Metropolitana } \\
\text { El. S. Paulo SA }\end{array}$ & Energía eléctrica & Brasil & 100 & 10238350 & - \\
\hline 36. & Light SA & Energía eléctrica & Brasil & 100 & 6305624 & 0,1156 \\
\hline 37. & Terna Participações S/A & Energía eléctrica & Brasil & 100 & 1863975 & - \\
\hline 38. & Tractebel Energia SA & Energía eléctrica & Brasil & 100 & 3657187 & 0,3025 \\
\hline 39. & Banco Bradesco SA & Finanzas y Seguros & Brasil & 100 & 755178 & 0,4624 \\
\hline 40. & Banco do Brasil SA & Finanzas y Seguros & Brasil & 100 & 17115018 & 0,1681 \\
\hline 41. & $\begin{array}{l}\text { Banco Itau Holding } \\
\text { Financeira SA }\end{array}$ & Finanzas y Seguros & Brasil & 100 & 27473530 & 0,5776 \\
\hline 42. & Banco Nossa Caixa SA & Finanzas y Seguros & Brasil & 100 & 2208829 & 0,0400 \\
\hline 43. & $\begin{array}{l}\text { Banestes S/A (Banco Estado } \\
\text { Espirito Santo) }\end{array}$ & Finanzas y Seguros & Brasil & 100 & 1312913 & 0,0529 \\
\hline 44. & Porto Seguro SA & Finanzas y Seguros & Brasil & 100 & 7465665 & 0,1156 \\
\hline 45. & $\begin{array}{l}\text { Unibanco (Uniao de Bancos } \\
\text { Brasileiros SA) }\end{array}$ & Finanzas y Seguros & Brasil & 100 & 15986789 & 0,5476 \\
\hline 46. & Weg SA & Maquinaria Industrial & Brasil & 100 & 2280562 & 0,1600 \\
\hline 47. & Eternit SA & Minerales no metálicos & Brasil & 100 & 1647539 & 0,2401 \\
\hline 48. & Cia. Vale do Rio Doce & Minería & Brasil & 100 & 28738772 & 0,3844 \\
\hline 49. & Bradespar SA & Otros & Brasil & 100 & 26184378 & 0,3136 \\
\hline 50. & $\begin{array}{l}\text { Cia. Saneamento Basico } \\
\text { Estado Sao Paulo }\end{array}$ & Otros & Brasil & 100 & 2150095 & 0,2916 \\
\hline 51. & $\begin{array}{l}\text { Cia. de Saneamento de } \\
\text { Minas Gerais }\end{array}$ & Otros & Brasil & 100 & 692202 & 0,0961 \\
\hline 52. & Contax Participacoes SA & Otros & Brasil & 100 & 1631399 & 0,0900 \\
\hline 53. & Csu Cardsystem S/A & Otros & Brasil & 100 & 552539 & - \\
\hline 54. & Diagnosticos da America SA & Otros & Brasil & 100 & 2877559 & 0,2601 \\
\hline 55. & Duratex SA & Otros & Brasil & 100 & 19675286 & 0,1936 \\
\hline 56. & Itausa (Investimentos Itau SA) & Otros & Brasil & 100 & 23456472 & 0,4761 \\
\hline 57. & Medial Saude S/A & Otros & Brasil & 100 & 14301564 & - \\
\hline 58. & $\begin{array}{l}\text { Net Servicos de } \\
\text { Comunicacao SA }\end{array}$ & Otros & Brasil & 100 & 30473820 & 0,2809 \\
\hline 59. & Odontoprev S/A & Otros & Brasil & 100 & 647867 & - \\
\hline 60. & $\begin{array}{l}\text { Sao Carlos Empreends e } \\
\text { Participacoes SA }\end{array}$ & Otros & Brasil & 100 & 3470248 & 0,0064 \\
\hline 61. & Saraiva SA Livreiros Editores & Otros & Brasil & 100 & 4882616 & 0,1369 \\
\hline 62. & Souza Cruz SA & Otros & Brasil & 100 & 2750910 & 0,1089 \\
\hline 63. & Vivax S/A & Otros & Brasil & 100 & 3194330 & 0,1936 \\
\hline 64. & Aracruz Celulose SA & Papel y Celulosa & Brasil & 100 & 8031581 & 0,3844 \\
\hline 65. & Klabin SA & Papel y Celulosa & Brasil & 100 & 5990128 & 0,3600 \\
\hline 66. & Suzano Papel e Celulose SA & Papel y Celulosa & Brasil & 100 & 13648883 & 0,3481 \\
\hline 67. & $\begin{array}{l}\text { Votorantim Celulose e } \\
\text { Papel SA }\end{array}$ & Papel y Celulosa & Brasil & 100 & 10136851 & 0,3969 \\
\hline 68. & $\begin{array}{l}\text { Cia. Brasileira de Petroleo } \\
\text { Ipiranga }\end{array}$ & Petróleo y Gas & Brasil & 100 & 2132619 & 0,3249 \\
\hline 69. & $\begin{array}{l}\text { Companhia de Gas de } \\
\text { Sao Paulo (Comgas) }\end{array}$ & Petróleo y Gas & Brasil & 100 & 2161000 & 0,1936 \\
\hline 70. & $\begin{array}{l}\text { Petroleo Brasileiro S/A } \\
\text { (Petrobras) }\end{array}$ & Petróleo y Gas & Brasil & 100 & 217798095 & 0,3025 \\
\hline 71. & Braskem SA & Química & Brasil & 100 & 8706350 & 0,2209 \\
\hline
\end{tabular}




\begin{tabular}{|c|c|c|c|c|c|c|}
\hline & Razón social & Sector económico & País sede & $\begin{array}{l}\text { Presencia } \\
(\%)\end{array}$ & $\begin{array}{l}\text { Volumen } \\
\text { transado } \\
\text { (dólares) }\end{array}$ & $\begin{array}{c}\mathrm{R}^{2} \text { respecto } \\
\text { del S\&P } 500 \\
\text { (cotizado en } \\
\text { dólares) }\end{array}$ \\
\hline 72. & $\begin{array}{l}\text { Copesul (Companhia } \\
\text { Petroquimica do Sul) }\end{array}$ & Química & Brasil & 100 & 2340576 & 0,2809 \\
\hline 73. & $\begin{array}{l}\text { Fertilizantes Fosfatados SA } \\
\text { (Fosfertil) }\end{array}$ & Química & Brasil & 100 & 571294 & 0,0576 \\
\hline 74. & MyG Poliester SA & Química & Brasil & 100 & 549651 & 0,0484 \\
\hline 75. & $\begin{array}{l}\text { Unipar (Uniao Inds. } \\
\text { Petroquimicas S/A) }\end{array}$ & Química & Brasil & 100 & 876661 & 0,2025 \\
\hline 76. & Acesita SA & Siderurgia y Metalurgia & Brasil & 100 & 1126587 & 0,1296 \\
\hline 77. & Arcelor Brasil SA & Siderurgia y Metalurgia & Brasil & 100 & 10015280 & 0,0961 \\
\hline 78. & $\begin{array}{l}\text { Companhia Siderurgica } \\
\text { Nacional }\end{array}$ & Siderurgia y Metalurgia & Brasil & 100 & 33202742 & 0,3249 \\
\hline 79. & Confab Industrial SA & Siderurgia y Metalurgia & Brasil & 100 & 569670 & 0,1521 \\
\hline 80. & Forjas Taurus SA & Siderurgia y Metalurgia & Brasil & 100 & 748561 & 0,0576 \\
\hline 81. & Gerdau SA & Siderurgia y Metalurgia & Brasil & 100 & 26101080 & 0,2916 \\
\hline 82. & Lupatech SA & Siderurgia y Metalurgia & Brasil & 100 & 5439072 & - \\
\hline 83. & Mangels Industrial SA & Siderurgia y Metalurgia & Brasil & 100 & 520828 & 0,0196 \\
\hline 84. & Metalurgica Gerdau SA & Siderurgia y Metalurgia & Brasil & 100 & 7717144 & 0,3136 \\
\hline 85. & Paranapanema SA & Siderurgia y Metalurgia & Brasil & 100 & 2441419 & 0,1849 \\
\hline 86. & $\begin{array}{l}\text { Usinas Siderurgicas de } \\
\text { Minas Gerais SA }\end{array}$ & Siderurgia y Metalurgia & Brasil & 100 & 38857661 & 0,3136 \\
\hline 87. & Totvs S/A & Software y Datos & Brasil & 100 & 2270803 & - \\
\hline 88. & Universo Online SA & Software y Datos & Brasil & 100 & 1461806 & 0,1369 \\
\hline 89. & $\begin{array}{l}\text { Brasil Telecom } \\
\text { Participacoes SA }\end{array}$ & Telecomunicación & Brasil & 100 & 9029130 & 0,3721 \\
\hline 90. & Brasil Telecom SA & Telecomunicación & Brasil & 100 & 13901411 & 0,2809 \\
\hline 91. & $\begin{array}{l}\text { Tele Norte Leste } \\
\text { Participações S/A }\end{array}$ & Telecomunicación & Brasil & 100 & 25721510 & 0,5329 \\
\hline 92. & $\begin{array}{l}\text { Telecomunicacoes de } \\
\text { Sao Paulo S/A (Telesp) }\end{array}$ & Telecomunicación & Brasil & 100 & 1607180 & 0,3364 \\
\hline 93. & Telemar Norte Leste S/A & Telecomunicación & Brasil & 100 & 5019651 & 0,4624 \\
\hline 94. & $\begin{array}{l}\text { Telemig Celular } \\
\text { Participacoes SA }\end{array}$ & Telecomunicación & Brasil & 100 & 878176 & 0,1521 \\
\hline 95. & Tim Participacoes SA & Telecomunicación & Brasil & 100 & 8328673 & 0,5041 \\
\hline 96. & Vivo Participacoes S/A & Telecomunicación & Brasil & 100 & 10543697 & 0,3721 \\
\hline 97. & Grendene SA & Textil & Brasil & 100 & 3960712 & 0,1024 \\
\hline 98. & Guararapes Confeccoes SA & Textil & Brasil & 100 & 2094705 & 0,1521 \\
\hline 99. & All-America Latina Logistica SA & Transporte y Servicios & Brasil & 100 & 39355876 & 0,1764 \\
\hline 100. & Cia. Docas de Imbituba & Transporte y Servicios & Brasil & 100 & 734898 & 0,0576 \\
\hline 101. & $\begin{array}{l}\text { Companhia de Concessoes } \\
\text { Rodoviarias }\end{array}$ & Transporte y Servicios & Brasil & 100 & 11376968 & 0,0729 \\
\hline 102. & $\begin{array}{l}\text { Gol Linhas Aéreas } \\
\text { Inteligentes S/A }\end{array}$ & Transporte y Servicios & Brasil & 100 & 6655735 & 0,4225 \\
\hline 103. & Localiza Rent a Car SA & Transporte y Servicios & Brasil & 100 & 16048650 & 0,2025 \\
\hline 104. & Obrascon Huarte Lain Brasil SA & Transporte y Servicios & Brasil & 100 & 718585 & 0,2601 \\
\hline 105. & Santos-Brasil SA & Transporte y Servicios & Brasil & 100 & 3641500 & - \\
\hline 106. & Tam SA & Transporte y Servicios & Brasil & 100 & 9264557 & 0,3481 \\
\hline 107. & $\begin{array}{l}\text { Embraer (Emp Brasileira } \\
\text { Aeronautica SA) }\end{array}$ & Vehículos y Piezas & Brasil & 100 & 12782519 & 0,2500 \\
\hline 108. & Fras-Le SA & Vehículos y Piezas & Brasil & 100 & 1512538 & 0,0676 \\
\hline 109. & Lochpe-Maxion SA & Vehículos y Piezas & Brasil & 100 & 2423422 & 0,0144 \\
\hline
\end{tabular}




\begin{tabular}{|c|c|c|c|c|c|c|}
\hline & Razón social & Sector económico & País sede & $\begin{array}{l}\text { Presencia } \\
(\%)\end{array}$ & $\begin{array}{l}\text { Volumen } \\
\text { transado } \\
\text { (dólares) }\end{array}$ & $\begin{array}{c}\mathrm{R}^{2} \text { respecto } \\
\text { del S\&P } 500 \\
\text { (cotizado en } \\
\text { dólares) } \\
\end{array}$ \\
\hline 110. & Marcopolo SA & Vehículos y Piezas & Brasil & 100 & 1300412 & 0,0900 \\
\hline 111. & $\begin{array}{l}\text { Plascar Participacoes } \\
\text { Industriais SA }\end{array}$ & Vehículos y Piezas & Brasil & 100 & 2788063 & 0,0324 \\
\hline 112. & $\begin{array}{l}\text { Randon SA S/A Implementos } \\
\text { e Participacoes }\end{array}$ & Vehículos y Piezas & Brasil & 100 & 2257243 & 0,1764 \\
\hline 113. & Masisa S. A. & Agro y Pesca & Chile & 100 & 9365158 & 0,1764 \\
\hline 114. & Embotelladora Andina S. A. & Alimentos y Bebidas & Chile & 100 & 668691 & 0,2500 \\
\hline 115. & Empresas lansa S. A. & Alimentos y Bebidas & Chile & 100 & 2302492 & 0,0529 \\
\hline 116. & Viña Concha y Toro S. A. & Alimentos y Bebidas & Chile & 100 & 1023422 & 0,0961 \\
\hline 117. & Cencosud S. A. & Comercio & Chile & 100 & 9644716 & 0,3364 \\
\hline 118. & $\begin{array}{l}\text { Distribución y Servicios } \\
\text { (DyS) S. A. }\end{array}$ & Comercio & Chile & 100 & 2132439 & 0,1936 \\
\hline 119. & Empresas La Polar S. A. & Comercio & Chile & 100 & 3207187 & 0,3249 \\
\hline 120. & Ripley Corp S. A. & Comercio & Chile & 100 & 1390744 & 0,3969 \\
\hline 121. & S.A.C.I. Falabella & Comercio & Chile & 100 & 4284804 & 0,1369 \\
\hline 122. & Aes Gener S. A. & Energía eléctrica & Chile & 100 & 1896515 & 0,0841 \\
\hline 123. & Colbun S. A. & Energía eléctrica & Chile & 100 & 743649 & 0,1444 \\
\hline 124. & $\begin{array}{l}\text { Compañía General de } \\
\text { Electricidad S. A. }\end{array}$ & Energía eléctrica & Chile & 100 & 641606 & 0,0841 \\
\hline 125. & $\begin{array}{l}\text { Empresa Nacional de } \\
\text { Electricidad S. A. }\end{array}$ & Energía eléctrica & Chile & 100 & 3180513 & 0,5041 \\
\hline 126. & Enersis S. A. & Energía eléctrica & Chile & 100 & 63 & 0,5929 \\
\hline 127. & Almendral S. A. & Finanzas y Seguros & Chile & 100 & 62 & 0,1936 \\
\hline 128. & AntarChile S. A.S.A. & Finanzas y Seguros & Chile & 100 & 1918768 & 0,1444 \\
\hline 129. & Banco Crédito e Inversiones & Finanzas y Seguros & Chile & 100 & 633341 & 0,3249 \\
\hline 130. & Banco de Chile & Finanzas y Seguros & Chile & 100 & 1577676 & 0,0729 \\
\hline 131. & Banco Santander-Chile & Finanzas y Seguros & Chile & 100 & 3331593 & 0,3364 \\
\hline 132. & Corpbanca & Finanzas y Seguros & Chile & 100 & 1873849 & 0,0900 \\
\hline 133. & $\begin{array}{l}\text { Inversiones Aguas } \\
\text { Metropolitanas S. A. }\end{array}$ & Finanzas y Seguros & Chile & 100 & 1649789 & 0,1849 \\
\hline 134. & Cementos Bío Bío S. A. & Minerales no metálicos & Chile & 100 & 861217 & 0,0361 \\
\hline 135. & $\begin{array}{l}\text { Sociedad Química y } \\
\text { Minera de Chile S. A. }\end{array}$ & Minería & Chile & 100 & 636916 & 0,2601 \\
\hline 136. & Forus S. A. & Otros & Chile & 100 & 782418 & - \\
\hline 137. & Paz Corp S. A. & Otros & Chile & 100 & 646553 & - \\
\hline 138. & Empresas CMPC S. A. & Papel y Celulosa & Chile & 100 & 2599753 & 0,2209 \\
\hline 139. & Empresas Copec S. A. & Petróleo y Gas & Chile & 100 & 3279118 & 0,1764 \\
\hline 140. & CAP S. A. & Siderurgia y Metalurgia & Chile & 100 & 39509 & 0,1225 \\
\hline 141. & Cintac S. A. & Siderurgia y Metalurgia & Chile & 100 & 1218152 & 0,0025 \\
\hline 142. & Madeco S. A. & Siderurgia y Metalurgia & Chile & 100 & 1791308 & 0,1681 \\
\hline 143. & Sonda S. A. & Software y Datos & Chile & 100 & 1448565 & - \\
\hline 144. & $\begin{array}{l}\text { Cía. de Telecomunicaciones } \\
\text { de Chile S. A. }\end{array}$ & Telecomunicación & Chile & 100 & 6392492 & 0,1600 \\
\hline 145. & $\begin{array}{l}\text { Empresa Nacional de } \\
\text { Teleacomunicaciones S. A. }\end{array}$ & Telecomunicación & Chile & 100 & 2223674 & 0,3600 \\
\hline 146. & $\begin{array}{l}\text { Compañía Sudamericana } \\
\text { de Vapores S. A. }\end{array}$ & Transporte y Servicios & Chile & 100 & 818064 & 0,1089 \\
\hline 147. & Lan Airlines S. A. & Transporte y Servicios & Chile & 100 & 6076116 & 0,3600 \\
\hline 148. & I.S.A. - Previligiadas & Energía eléctrica & Colombia & 100 & 3318465 & 0,3364 \\
\hline
\end{tabular}




\begin{tabular}{|c|c|c|c|c|c|c|}
\hline & Razón social & Sector económico & País sede & $\begin{array}{l}\text { Presencia } \\
\text { (\%) }\end{array}$ & $\begin{array}{l}\text { Volumen } \\
\text { transado } \\
\text { (dólares) }\end{array}$ & $\begin{array}{c}\mathrm{R}^{2} \text { respecto } \\
\text { del S\&P } 500 \\
\text { (cotizado en } \\
\text { dólares) }\end{array}$ \\
\hline 149. & $\begin{array}{l}\text { Cía. Colombiana de } \\
\text { Inversiones }\end{array}$ & Finanzas y Seguros & Colombia & 100 & 969551 & 0,2304 \\
\hline 150. & $\begin{array}{l}\text { Cía. Suramericana de } \\
\text { Inversiones }\end{array}$ & Finanzas y Seguros & Colombia & 100 & 4825170 & 0,3025 \\
\hline 151 & $\begin{array}{l}\text { Corporación Financiera } \\
\text { Colombiana S. A. }\end{array}$ & Finanzas y Seguros & Colombia & 100 & 12498973 & 0,2116 \\
\hline 152 & Inversiones Argos S. A. & Finanzas y Seguros & Colombia & 100 & 1574458 & 0,2704 \\
\hline 153 & Cementos Argos S. A. & Minerales no metálicos & Colombia & 100 & 2328403 & 0,2304 \\
\hline 154 & $\begin{array}{l}\text { Fábrica de Hilados y } \\
\text { Tejidos del Hato }\end{array}$ & Textil & Colombia & 100 & 523846 & 0,0784 \\
\hline 155 & Alsea, S. A. B. de C.V. & Alimentos y Bebidas & México & 100 & 1308696 & 0,1521 \\
\hline 156. & $\begin{array}{l}\text { Coca Cola Femsa, } \\
\text { S. A. de C.V. }\end{array}$ & Alimentos y Bebidas & México & 100 & 2668812 & 0,1764 \\
\hline 157. & $\begin{array}{l}\text { Embotelladoras Arca, } \\
\text { S.A. de C.V. }\end{array}$ & Alimentos y Bebidas & México & 100 & 1808380 & 0,1444 \\
\hline 158. & $\begin{array}{l}\text { Fomento Económico } \\
\text { Mexicano, S. A. de C.V. }\end{array}$ & Alimentos y Bebidas & México & 100 & 9773299 & 0,4624 \\
\hline 159. & Gruma, S. A. de C.V. & Alimentos y Bebidas & México & 100 & 8448891 & 0,2500 \\
\hline 160. & Grupo Bimbo, S. A. de C.V. & Alimentos y Bebidas & México & 100 & 1397322 & 0,1156 \\
\hline 161. & Grupo Modelo, S. A. de C.V.. & Alimentos y Bebidas & México & 100 & 7190253 & 0,3249 \\
\hline 162. & $\begin{array}{l}\text { Controladora Comercial } \\
\text { Mexicana, S. A. B. de C. V. }\end{array}$ & Comercio & México & 100 & 6350277 & 0,1764 \\
\hline 163. & Grupo Famsa, S. A. de C.V. & Comercio & México & 100 & 1593559 & - \\
\hline 164. & $\begin{array}{l}\text { Organización Soriana, } \\
\text { S.A. de C.V. }\end{array}$ & Comercio & México & 100 & 7203218 & 0,1296 \\
\hline 165. & $\begin{array}{l}\text { Wal-Mart de México, } \\
\text { S.A. de C.V. }\end{array}$ & Comercio & México & 100 & 77414634 & 0,1764 \\
\hline 166. & $\begin{array}{l}\text { Carso Infraestructura y } \\
\text { Construcción, S. A. de C. V. }\end{array}$ & Construcción & México & 100 & 2846771 & 0,0529 \\
\hline 167. & Consorcio ARA, S. A. de C.V. & Construcción & México & 100 & 4931838 & 0,2809 \\
\hline 168. & Corporación Geo, S. A. de C. V. & Construcción & México & 100 & 28050610 & 0,1600 \\
\hline 169. & $\begin{array}{l}\text { Desarrolladora Homex, } \\
\text { S. A. de C.V. }\end{array}$ & Construcción & México & 100 & 6972275 & 0,5476 \\
\hline 170. & Empresas ICA, S. A. de C.V. & Construcción & México & 100 & 269 & 0,1521 \\
\hline 171. & $\begin{array}{l}\text { Impulsora del Desarrollo y } \\
\text { el Empleo en América Latina, } \\
\text { S. A. de C.V. }\end{array}$ & Construcción & México & 100 & 11283411 & 0,0016 \\
\hline 172. & $\begin{array}{l}\text { Promotora y Operadora de } \\
\text { Infraestructura, S. A. de C.V. }\end{array}$ & Construcción & México & 100 & 969092 & 0,0225 \\
\hline 173. & Sare Holding, S. A. B. de C. V. & Construcción & México & 100 & 2020426 & 0,1849 \\
\hline 174. & $\begin{array}{l}\text { Urbi Desarrollos Urbanos, } \\
\text { S.A. de C.V. }\end{array}$ & Construcción & México & 100 & 5513451 & 0,2209 \\
\hline 175. & $\begin{array}{l}\text { Grupo Financiero Banorte, } \\
\text { S.A. de C. V. }\end{array}$ & Finanzas y Seguros & México & 100 & 15059682 & 0,3481 \\
\hline 176. & $\begin{array}{l}\text { Grupo Financiero Inbursa, } \\
\text { S.A. de C.V. }\end{array}$ & Finanzas y Seguros & México & 100 & 726190 & 0,0100 \\
\hline 177. & Cemex, S. A. de C.V. & Minerales no metálicos & México & 100 & 43481958 & 0,4624 \\
\hline 178. & $\begin{array}{l}\text { Grupo México, S. A. B. } \\
\text { de C.V. }\end{array}$ & Minería & México & 100 & 29197726 & 0,3249 \\
\hline
\end{tabular}




\begin{tabular}{|c|c|c|c|c|c|c|}
\hline & Razón social & Sector económico & País sede & $\begin{array}{c}\text { Presencia } \\
(\%)\end{array}$ & $\begin{array}{l}\text { Volumen } \\
\text { transado } \\
\text { (dólares) }\end{array}$ & $\begin{array}{c}\mathrm{R}^{2} \text { respecto } \\
\text { del S\&P } 500 \\
\text { (cotizado en } \\
\text { dólares) }\end{array}$ \\
\hline 179. & $\begin{array}{l}\text { Industrias Peñoles, S. A. } \\
\text { de C. V. }\end{array}$ & Minería & México & 100 & 1285296 & 0,0676 \\
\hline 180. & Grupo Carso, S. A. de C. V. & Otros & México & 100 & 2616097 & 0,2500 \\
\hline 181. & Grupo Televisa, S. A. de C. V. & Otros & México & 100 & 19644757 & 0,3969 \\
\hline 182. & TV Azteca, S. A. de C. V. & Otros & México & 100 & 1587589 & 0,0961 \\
\hline 183. & $\begin{array}{l}\text { Kimberly-Clark de México, } \\
\text { S.A. de C.V. }\end{array}$ & Papel y Celulosa & México & 100 & 2375533 & 0,4225 \\
\hline 184. & Mexichem, S.A. de C.V. & Química & México & 100 & 798568 & 0,1444 \\
\hline 185. & Alfa, S. A. de C.V. & Siderurgia y Metalurgia & México & 100 & 3779968 & 0,1600 \\
\hline 186. & Grupo Simec, S. A. B. de C. V. & Siderurgia y Metalurgia & México & 100 & 12967231 & 0,1369 \\
\hline 187. & Industrias $\mathrm{CH}, \mathrm{S}$. A. de C.V. & Siderurgia y Metalurgia & México & 100 & 5282241 & 0,0529 \\
\hline 188. & América Móvil, S. A. de C.V. & Telecomunicación & México & 100 & 161209897 & 0,3721 \\
\hline 189. & $\begin{array}{l}\text { Carso Global Telecom, } \\
\text { S. A. de C.V. }\end{array}$ & Telecomunicación & México & 100 & 3223766 & 0,1225 \\
\hline 190. & $\begin{array}{l}\text { Teléfonos de México, } \\
\text { S. A. de C.V. }\end{array}$ & Telecomunicación & México & 100 & 18740775 & 0,1521 \\
\hline 191. & Cintra, S. A. de C. V. & Transporte y Servicios & México & 100 & 1014999 & 0,1369 \\
\hline 192. & $\begin{array}{l}\text { Grupo Aeroportuario del } \\
\text { Centro Norte, S. A. B. de C. V. }\end{array}$ & Transporte y Servicios & México & 100 & 1250587 & - \\
\hline 193. & $\begin{array}{l}\text { Grupo Aeroportuario del } \\
\text { Pacífico }\end{array}$ & Transporte y Servicios & México & 100 & 4812657 & - \\
\hline 194. & $\begin{array}{l}\text { Grupo Aeroportuario, } \\
\text { S.A. de C.V. }\end{array}$ & Transporte y Servicios & México & 100 & 1599487 & 0,2500 \\
\hline 195. & Banco Continental & Finanzas y Seguros & Perú & 100 & 4113353 & 0,0064 \\
\hline 196. & $\begin{array}{l}\text { Compañía Minera } \\
\text { Atacocha S. A. }\end{array}$ & Minería & Perú & 100 & 1130752 & 0,0049 \\
\hline 197. & Minsur S. A. & Minería & Perú & 100 & 2824706 & 0,0009 \\
\hline 198. & Peru Copper Inc. & Minería & Perú & 100 & 695295 & 0,0004 \\
\hline 199. & $\begin{array}{l}\text { Sociedad Minera Cerro } \\
\text { Verde S. A. A. }\end{array}$ & Minería & Perú & 100 & 617012 & 0,0144 \\
\hline 200. & Vena Resources Inc. & Minería & Perú & 100 & 779986 & - \\
\hline 201. & $\begin{array}{l}\text { Volcan Compañía } \\
\text { Minera S. A. A. }\end{array}$ & Minería & Perú & 100 & 2698106 & 0,0196 \\
\hline 202. & $\begin{array}{l}\text { Empresa Siderúrgica del } \\
\text { Perú S. A. A. }\end{array}$ & Siderurgia y Metalurgia & Perú & 100 & 758550 & - \\
\hline
\end{tabular}

Fuente: Economática. 


\section{Anexo 2 \\ Empresas emisoras de ADR en América Latina}

\begin{tabular}{|c|c|c|}
\hline Razón social & País sede & Sector económico \\
\hline 1. Quilmes Industrial & Argentina & Alimentos y Bebidas \\
\hline 2. BBVA Banco Francés S. A. & Argentina & Finanzas y Seguros \\
\hline 3. Banco Macro Bansud S. A. & Argentina & Finanzas y Seguros \\
\hline 4. Nortel Inversora S. A. & Argentina & Otros \\
\hline 5. Petrobrás Energía Participaciones S. A. & Argentina & Otros \\
\hline 6. IRSA Inversiones y Representaciones S. A. & Argentina & Otros \\
\hline 7. MetroGas S. A. & Argentina & Petróleo y Gas \\
\hline 8. Transportadora de Gas del Sur S.A. & Argentina & Petróleo y Gas \\
\hline 9. YPF S.A. & Argentina & Petróleo y Gas \\
\hline 10. Ternium & Argentina & Siderurgia y Metalurgia \\
\hline 11. Tenaris S. A. & Argentina & Siderurgia y Metalurgia \\
\hline 12. Tenaris Siderca S. A. & Argentina & Siderurgia y Metalurgia \\
\hline 13. Telefónica de Argentina S. A. & Argentina & Telecomunicación \\
\hline 14. Telecom Argentina S. A. & Argentina & Telecomunicación \\
\hline 15. Perdigao SA & Brasil & Alimentos y Bebidas \\
\hline 16. Sadia SA & Brasil & Alimentos y Bebidas \\
\hline 17. Companhia de Bebidas Das Americas (Ambev) & Brasil & Alimentos y Bebidas \\
\hline 18. Companhia de Bebidas Das Americas (Ambev) & Brasil & Alimentos y Bebidas \\
\hline 19. Perdigao SA & Brasil & Alimentos y Bebidas \\
\hline 20. Companhia Brasileira de Distribuicao & Brasil & Comercio \\
\hline 21. CPFL Energia SA & Brasil & Energía eléctrica \\
\hline 22. Cia. Paranaense de Energia (Copel) & Brasil & Energía eléctrica \\
\hline 23. Companhia Energetica de Minas Gerais & Brasil & Energía eléctrica \\
\hline 24. Banco Itau Holding Financeira SA & Brasil & Finanzas y Seguros \\
\hline 25. Banco Bradesco SA & Brasil & Finanzas y Seguros \\
\hline 26. Unibanco (Uniao de Bancos Brasileiros SA) & Brasil & Finanzas y Seguros \\
\hline 27. Cia. Vale do Rio Doce & Brasil & Minería \\
\hline 28. Cia. Vale do Rio Doce & Brasil & Minería \\
\hline 29. Cia. Saneamento Basico Estado Sao Paulo & Brasil & Otros \\
\hline 30. Votorantim Celulose e Papel SA & Brasil & Papel y Celulosa \\
\hline 31. Aracruz Celulose SA & Brasil & Papel y Celulosa \\
\hline 32. Petroleo Brasileiro S/A (Petrobras) & Brasil & Petróleo y Gas \\
\hline 33. Petroleo Brasileiro S/A (Petrobras) & Brasil & Petróleo y Gas \\
\hline 34. Ultrapar Participacoes SA & Brasil & Química \\
\hline 35. Braskem SA & Brasil & Química \\
\hline 36. Companhia Siderurgica Nacional & Brasil & Siderurgia y Metalurgia \\
\hline 37. Gerdau SA & Brasil & Siderurgia y Metalurgia \\
\hline 38. Embratel Participacoes SA & Brasil & Telecomunicación \\
\hline 39. Tele Norte Celular Participacoes SA & Brasil & Telecomunicación \\
\hline 40. Telemig Celular Participacoes SA & Brasil & Telecomunicación \\
\hline 41. Brasil Telecom SA & Brasil & Telecomunicación \\
\hline 42. Telecomunicacoes de Sao Paulo S/A (Telesp) & Brasil & Telecomunicación \\
\hline 43. Vivo Participacoes S/A & Brasil & Telecomunicación \\
\hline 44. Brasil Telecom Participacoes SA & Brasil & Telecomunicación \\
\hline 45. Tim Participacoes SA & Brasil & Telecomunicación \\
\hline 46. Tele Norte Leste Participações S/A & Brasil & Telecomunicación \\
\hline 47. Tele Sudeste Celular Participacoes SA & Brasil & Telecomunicación \\
\hline 48. Tele Leste Celular Participacoes SA & Brasil & Telecomunicación \\
\hline 49. Tele Nordeste Celular Participacoes S/A & Brasil & Telecomunicación \\
\hline 50. Tco Celular Participacoes S/A & Brasil & Telecomunicación \\
\hline
\end{tabular}




\begin{tabular}{|c|c|c|}
\hline Razón social & País sede & Sector económico \\
\hline 51. Telecomunicacoes Brasileiras S/A (celebras) & Brasil & Telecomunicación \\
\hline 52. Tam SA & Brasil & Transporte y Servicios \\
\hline 53. Gol Linhas Aéreas Inteligentes S/A & Brasil & Transporte y Servicios \\
\hline 54. Embraer (Emp Brasileira Aeronautica SA) & Brasil & Vehículos y Piezas \\
\hline 55. Embraer (Emp Brasileira Aeronautica SA) & Brasil & Vehículos y Piezas \\
\hline 56. Supermercados Unimarc S. A. & Chile & Agro y Pesca \\
\hline 57. Masisa S. A. & Chile & Agro y Pesca \\
\hline 58. Compañía Cervecerías Unidas S.A. & Chile & Alimentos y Bebidas \\
\hline 59. Embotelladora Andina S. A. & Chile & Alimentos y Bebidas \\
\hline 60. Embotelladora Andina S. A. & Chile & Alimentos y Bebidas \\
\hline 61. Viña Concha y Toro S. A. & Chile & Alimentos y Bebidas \\
\hline 62. Sta. Isabel & Chile & Comercio \\
\hline 63. Distribución y Servicio S. A. (DyS S. A.) & Chile & Comercio \\
\hline 64. Enersis S. A. & Chile & Energía eléctrica \\
\hline 65. Empresa Nacional de Electricidad S. A. & Chile & Energía eléctrica \\
\hline 66. Banco Bilbao Vizcaya Argentaria-Chile & Chile & Finanzas y Seguros \\
\hline 67. Banco de Chile & Chile & Finanzas y Seguros \\
\hline 68. Banco Santander-Chile & Chile & Finanzas y Seguros \\
\hline 69. Edwards & Chile & Finanzas y Seguros \\
\hline 70. Quinenco S.A. & Chile & Fondos \\
\hline 71. A. F. P. Provida S. A. & Chile & Fondos \\
\hline 72. Cristalerías Unidas de Chile S. A. & Chile & Minerales no metálicos \\
\hline 73. Sociedad Química y Minera de Chile S. A. & Chile & Minería \\
\hline 74. Sociedad Química y Minera de Chile S. A. & Chile & Minería \\
\hline 75. Exmasisa & Chile & Otros \\
\hline 76. Labchile & Chile & Química \\
\hline 77. Madeco S. A. & Chile & Siderurgia y Metalurgia \\
\hline 78. Cía. de Telecomunicaciones de Chile S.A. & Chile & Telecomunicación \\
\hline 79. Telex & Chile & Telecomunicación \\
\hline 80. Lan Airlines S. A. & Chile & Transporte y Servicios \\
\hline 81. Banco Colombia & Colombia & Finanzas y Seguros \\
\hline 82. Industrias Bachoco, S. A. de C.V. & México & Agro y Pesca \\
\hline 83. Savia, S. A. de C.V. & México & Agro y Pesca \\
\hline 84. Grupo Industrial Maseca, S. A. de C.V. & México & Alimentos y Bebidas \\
\hline 85. Coca Cola Femsa, S. A. de C.V. & México & Alimentos y Bebidas \\
\hline 86. Fomento Económico Mexicano, S. A. de C.V. & México & Alimentos y Bebidas \\
\hline 87. Pepsi Gemex, S. A. de C. V. & México & Alimentos y Bebidas \\
\hline 88. Panamco México, S. A. de C.V. & México & Alimentos y Bebidas \\
\hline 89. Grupo Casa Saba, S.A. de C.V. & México & Comercio \\
\hline 90. Controladora Comercial Mexicana, S. A. B. de C. V. & México & Comercio \\
\hline 91. Grupo Elektra, S. A. de C. V. & México & Comercio \\
\hline 92. Empresas ICA, S. A. de C.V. & México & Construcción \\
\hline 93. Desarrolladora Homex, S.A. de C.V. & México & Construcción \\
\hline 94. DESC, S.A. de C.V. & México & Maquinaria Industrial \\
\hline 95. Internacional de Cerámica, S. A. de C.V. & México & Minerales no metálicos \\
\hline 96. Vitro, S. A. de C.V. & México & Minerales no metálicos \\
\hline 97. Cemex, S.A. de C.V. & México & Minerales no metálicos \\
\hline 98. Grupo Radio Centro, S. A. de C.V. & México & Otros \\
\hline 99. TV Azteca, S. A. de C. V. & México & Otros \\
\hline 100. Grupo Televisa, S. A. de C.V. & México & Otros \\
\hline 101. Corporación Durango, S. A. de C.V. & México & Papel y Celulosa \\
\hline 102. Grupo IMSA, S. A. de C. V. & México & Siderurgia y Metalurgia \\
\hline 103. Grupo Lusacell, S. A. de C.V. & México & Telecomunicación \\
\hline
\end{tabular}




\begin{tabular}{|c|c|c|}
\hline Razón social & País sede & Sector económico \\
\hline 104. Teléfonos de México, S. A. de C.V. & México & Telecomunicación \\
\hline 105. América Móvil, S. A. de C. V. & México & Telecomunicación \\
\hline 106. Grupo TMM, S. A. de C. V. & México & Transporte y Servicios \\
\hline 107. Grupo Aeroportuario, S. A. de C.V. & México & Transporte y Servicios \\
\hline 108. Grupo Aeroportuario del Pacífico & México & Transporte y Servicios \\
\hline 109. ScotiaBank Perú S. A. A. & Perú & Finanzas y Seguros \\
\hline 110. Cía. de Minas Buenaventura S. A. A. & Perú & Minería \\
\hline 111. Credicorp Ltd. & Perú & Otros \\
\hline 112. Telefónica del Perú S.A.A. & Perú & Telecomunicación \\
\hline $\begin{array}{l}\text { 113. Compañía Anónima Nacional de } \\
\text { Teléfonos de Venezuela, C. A. }\end{array}$ & Venezuela & Telecomunicación \\
\hline
\end{tabular}

Fuente: Economática. 


\section{Anexo 3 \\ Empresas de América Latina con ADR en la NYSE que pasaron el filtro de liquidez}

\begin{tabular}{|c|c|c|c|c|}
\hline Razón social & Sector económico & País sede & $\begin{array}{l}\text { Presencia } \\
(\%)\end{array}$ & $\begin{array}{l}\text { Volumen } \\
\text { transado } \\
\text { (dólares) }\end{array}$ \\
\hline 1. Quilmes & Alimentos y Bebidas & Argentina & 100 & 1524030 \\
\hline 2. Banco Macro Bansud S. A. & Finanzas y Seguros & Argentina & 100 & 5452800 \\
\hline 3. BBVA Banco Francés S. A. & Finanzas y Seguros & Argentina & 100 & 1104985 \\
\hline 4. IRSA (Inversiones y Representaciones S. A.) & Otros & Argentina & 100 & 2008100 \\
\hline 5. Petrobrás Energia Participaciones S. A. & Otros & Argentina & 100 & 1493865 \\
\hline 6. Tenaris S. A. & Siderurgia y Metalurgia & Argentina & 100 & 63576071 \\
\hline 7. Ternium & Siderurgia y Metalurgia & Argentina & 100 & 3906495 \\
\hline 8. Telecom Argentina S. A. & Telecomunicación & Argentina & 100 & 3968002 \\
\hline 9. Telefónica de Argentina S. A. & Telecomunicación & Argentina & 100 & 910430 \\
\hline $\begin{array}{l}\text { 10. Companhia de Bebidas Das } \\
\text { Americas (Ambev) }\end{array}$ & Alimentos y Bebidas & Brasil & 100 & 27787152 \\
\hline 11. Perdigao SA & Alimentos y Bebidas & Brasil & 100 & 3840 \\
\hline 12. Sadia SA & Alimentos & & 100 & 3530 \\
\hline asileira de Distribuicao & Comercio & Brasil & 100 & 5643686 \\
\hline 14. Companhia $\mathrm{E}$ & Energía eléctrica & Brasil & 100 & 17212824 \\
\hline 15. Cia. Paranaense de Energia (Copel) & Energía eléctrica & Brasil & 100 & 3673004 \\
\hline 16. CPFL Energ & Energía eléctrica & Brasil & 100 & 3187032 \\
\hline 17. Banco Itau Holding Financeira SA & Finanzas y Seguros & Brasil & 100 & 50745123 \\
\hline 18. Banco Bradesco SA & Finanzas y Seguros & Brasil & 100 & 79292565 \\
\hline 19. Unibanco (Uniao de Bancos Brasileiros SA) & Finanzas y Seguros & Brasil & 100 & 81297882 \\
\hline 20. Cia. Vale & Minería & & 1001 & 185563425 \\
\hline asico Estado Sao Paulo & Otros & Brasil & 100 & 8043350 \\
\hline 22. Aracruz $\mathrm{Ce}$ & y Celul & Brasil & 100 & 19187014 \\
\hline 23. Voto & y Celulosa & Brasil & 100 & 7349390 \\
\hline 24. Petroleo Brasileiro S/A (Petrobras) & Petróleo y Gas & Brasil & 1001 & 190480695 \\
\hline 25. Braskem SA & Química & Brasil & 100 & 3852206 \\
\hline 26. Gerdau SA & Siderurgia y Metalurgia & Brasil & 100 & 24366168 \\
\hline 27. Companhia Siderurgica Nacional & Siderurgia y Metalurgia & Brasil & 100 & 35345667 \\
\hline 28. Bras & ón & & 100 & 4637 \\
\hline 29. Bras & Tel & $\mathrm{Br}$ & 100 & 7000 \\
\hline 30. Tele I & cación & Bras & 100 & 24940475 \\
\hline „lar Participacoes SA. & Telecomunicación & Brasil & 100 & 551608 \\
\hline pes de Sao Paulo S/A (Telesp) & Telecomunicación & Brasil & 100 & 1783733 \\
\hline 33. Tim Participacoes SA & Telecomunicación & Brasil & 100 & 10376493 \\
\hline 34. Vivo Participacoes S/A & Telecomunicación & Brasil & 100 & 10640505 \\
\hline 35. Gol Linhas Aéreas Inteligentes S/A & Transporte y Servicios & Brasil & 100 & 18662992 \\
\hline 36. Tam SA & Transporte y Servicios & Brasil & 100 & 10177650 \\
\hline 37. Embraer (Emp Brasileira Aeronautica SA) & Vehículos y Piezas & Brasil & 100 & 47836981 \\
\hline 38. Masisa & Agro y Pesca & Chile & 100 & 6072192 \\
\hline 39. Compañía Cervecerías & Alimentos y Bebidas & Chile & 100 & 549054 \\
\hline Nacional de Electricidad S. A. & Energía eléctrica & Chile & 100 & 790725 \\
\hline 41. Enersis S. A. & Energía eléctrica & Chile & 100 & 7805966 \\
\hline 42. Banco Santander-Chile & Finanzas y Seguros & Chile & 100 & 7285026 \\
\hline 43. Banco de Chile & Finanzas y Seguros & Chile & 100 & 2308371 \\
\hline 44. Sociedad Químic & Minería & Chile & 100 & 3885120 \\
\hline 45. Cía. de Telecomunicaciones de Chile S.A. & Telecomunicación & Chile & 100 & 4797045 \\
\hline
\end{tabular}




\section{Razón social}

Sector económico

País sede

Presencia

(\%)

Volumen

transado

(dólares)

46. Lan Airlines S. A.

Transporte y Servicios

Finanzas y Seguros

Alimentos y Bebidas

Alimentos y Bebidas

Chile

$100 \quad 8028288$

47. Banco Colombia

Colombia

100

8171628

48. Coca Cola Femsa, S. A. de C. V.

49. Fomento Económico Mexicano, S. A. de C.V.

50. Desarrolladora Homex, S. A. de C.V.

51. Cemex, S. A. de C.V.

52. Grupo Televisa, S. A. de C.V.

53. América Móvil, S. A. de C.V.

54. Teléfonos de México, S. A. de C.V.

55. Grupo Aeroportuario, S. A. de C. V.

56. Grupo Aeroportuario del Pacifico

57. Grupo TMM, S. A. de C.V.

58. Cía. de Minas Buenaventura S. A. A.

59. Credicorp Ltd.

60. Compañía Anónima Nacional de

México $\quad 100 \quad 7241488$

México $\quad 100 \quad 40063920$

$\begin{array}{ccrr}\text { Construcción } & \text { México } & 100 & 27062442 \\ \text { Minerales no metálicos } & \text { México } & 100 & 79405776 \\ \text { Otros } & \text { México } & 100 & 47807421 \\ \text { Telecomunicación } & \text { México } & 100 & 326177280 \\ \text { Telecomunicación } & \text { México } & 100 & 24871410 \\ \text { Transporte y Servicios } & \text { México } & 100 & 3632580 \\ \text { Transporte y Servicios } & \text { México } & 100 & 6413742 \\ \text { Transporte y Servicios } & \text { México } & 100 & 567854 \\ \text { Minería } & \text { Perú } & 100 & 10431428 \\ \text { Otros } & \text { Perú } & 100 & 30504105 \\ \text { Telecomunicación } & \text { Venezuela } & 100 & 34990568 \\ & & & \end{array}$

Fuente: Economática. 


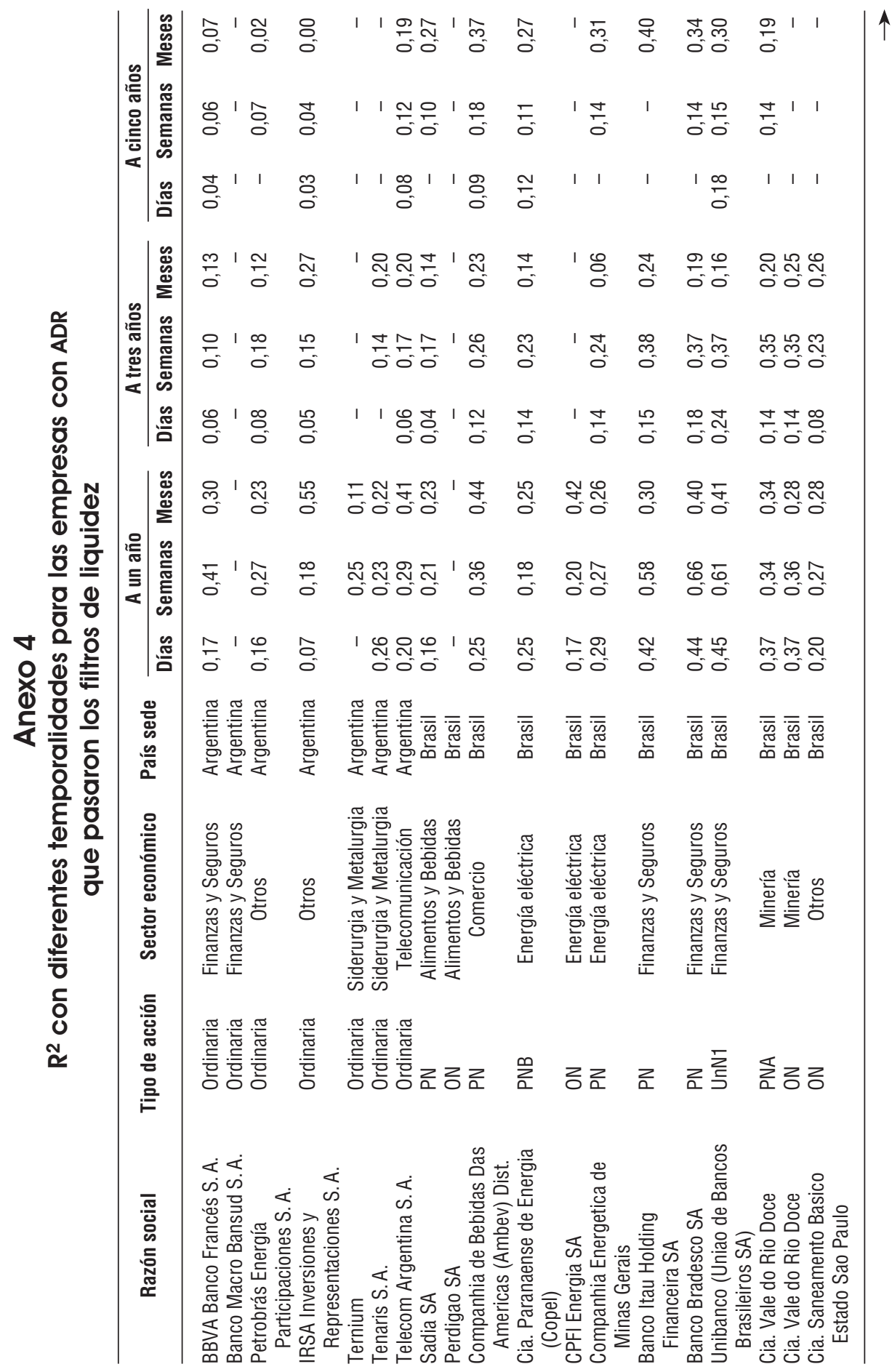




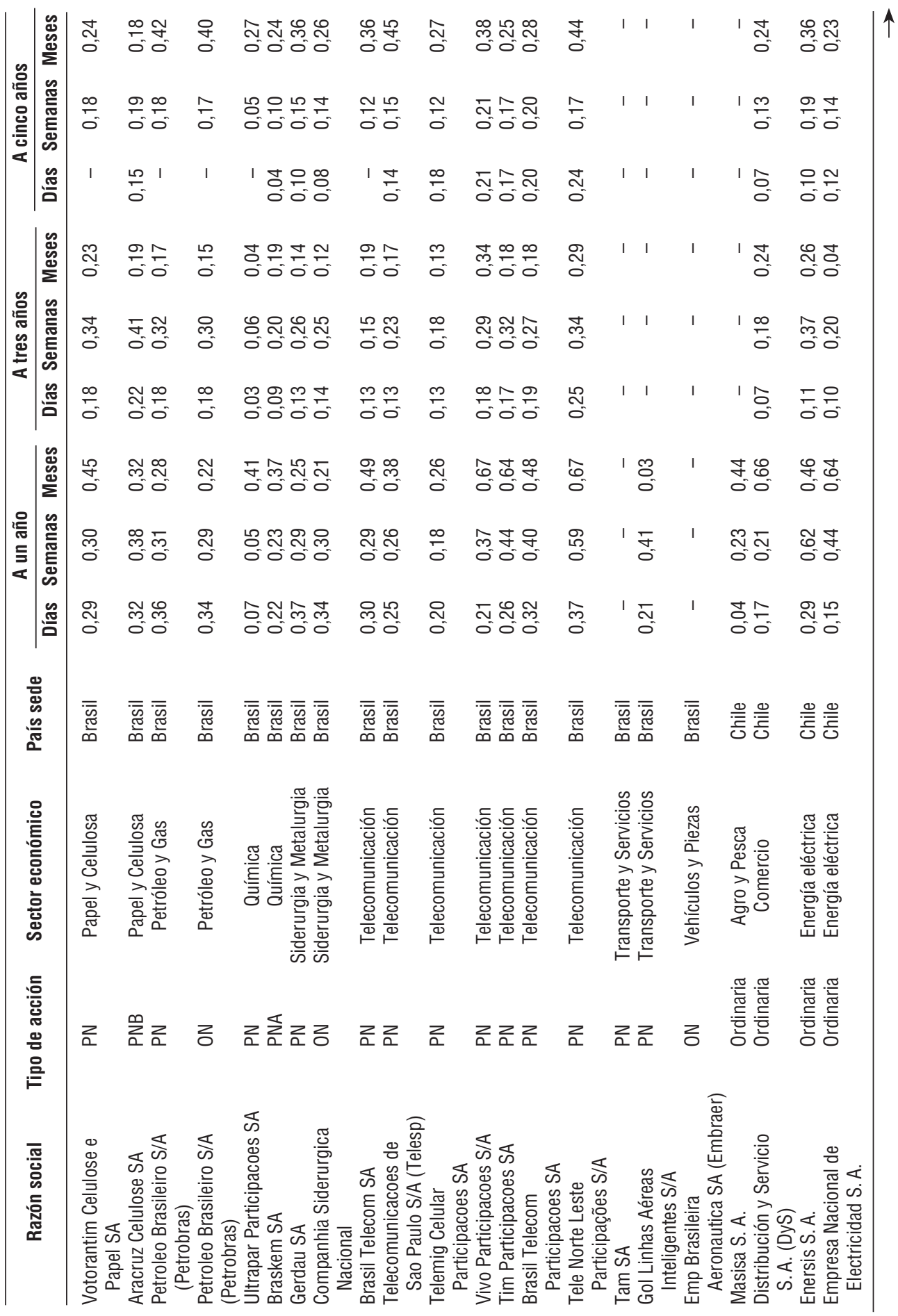




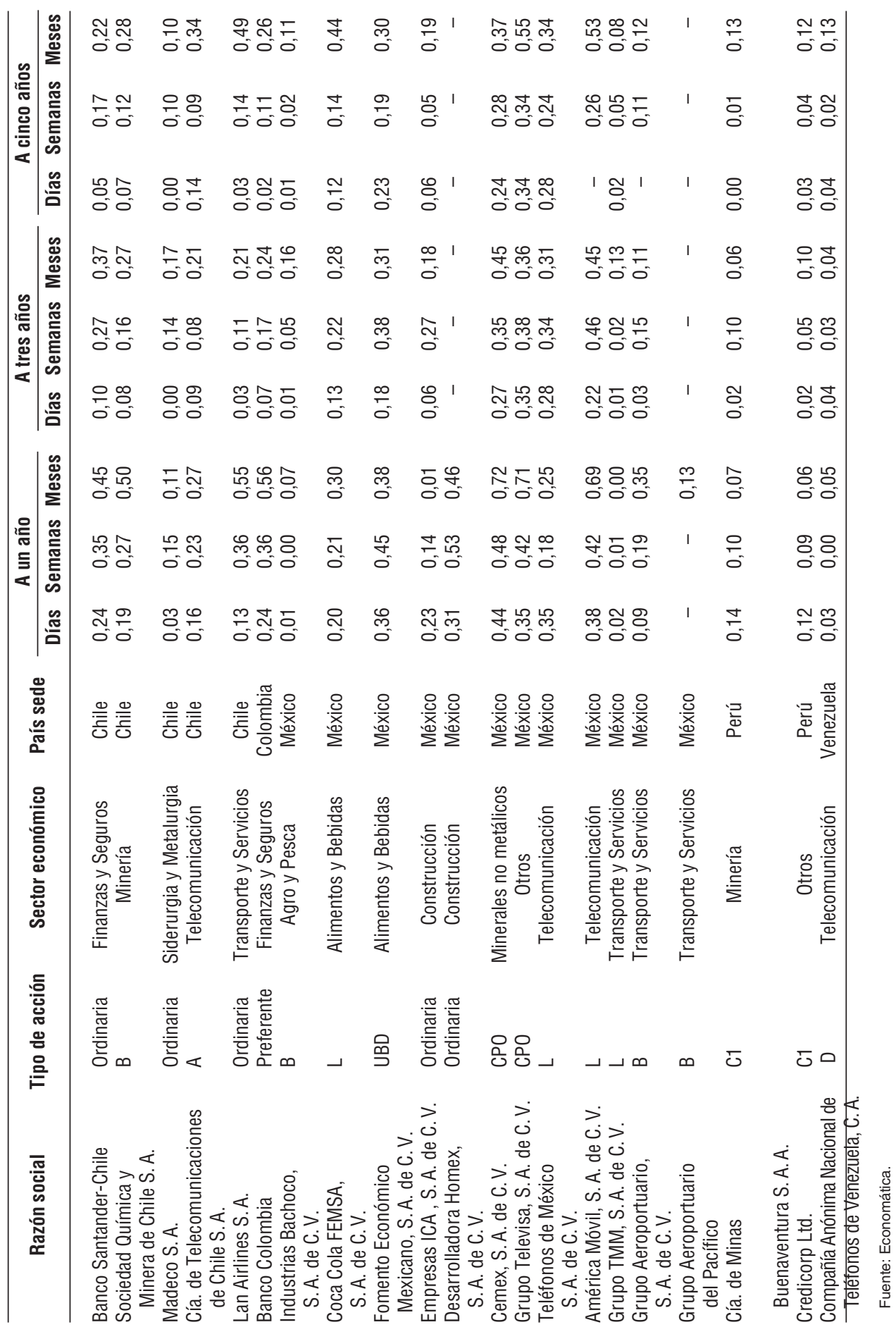




\section{Anexo 5 \\ Método utilizado para comparar $\mathrm{R}^{2}$ con diferentes horizontes temporales}

Se compararon nueve columnas de las cotizaciones de ADR con respecto del índice S\&P 500 con diferentes horizontes temporales utilizando el $\mathrm{R}^{2}$. Se buscaba comprobar cuál de las columnas presentaba valores más altos. Para comparar las columnas se decidió utilizar la función lógica conocida como «Si» (“If”). El cálculo se realizó con fecha de cierre al 9 de febrero del año 2007.

Esta función devuelve un valor si la condición especificada es VERDADERA y otro distinto si ese argumento es FALSO. La condición utilizada para la comparación fue si el valor de una columna era mayor que el de la otra (el $\mathrm{R}^{2}$ de una empresa con distintos horizontes temporales).

Para el periodo de un año, comparando los valores de las columnas (anexo 4), se obtienen los siguientes resultados:

- El cálculo por semanas es mayor que el de días en el $66,7 \%$ de los casos.

- El cálculo por meses es mayor que el de días en el 64,9\% de los casos.

- El cálculo por semanas es mayor que el de meses en el 57,9\% de los casos.

Por tanto, para el caso de un año se puede afirmar que el cálculo en semanas es aquel más representativo.

Para el periodo de tres años, al comparar los valores de las columnas se obtienen los siguientes resultados:

- El cálculo por semanas es mayor que el de días en el 96,1\% de los casos.

- El cálculo por meses es mayor que el de días en el 78,8\% de los casos.

- El cálculo en semanas es mayor que el de meses en el 59,6\% de los casos.

Por tanto, para el caso de tres años se puede afirmar que el cálculo en semanas es el más representativo.

Para el periodo de cinco años, comparando los valores de las columnas se obtienen los siguientes resultados:

- El cálculo en semanas es mayor que el de días en el $64,8 \%$ de los casos.

- El cálculo en meses es mayor que el de días en el 97,3\% de los casos.

- El cálculo en meses es mayor que el de semanas en el 90,0\% de los casos.

Por tanto, para el caso de cinco años se puede afirmar que el cálculo en meses es el más representativo. 
Definidos estos parámetros, se procedió a comparar las tres series registradas como más representativas para establecer cuál poseía la mayor representatividad. El resultado de la comparación es el siguiente:

- El cálculo de un año en semanas es mayor que el de tres años en semanas en el 74,1\% de los casos.

- El cálculo de un año en semanas es mayor que el de cinco años en meses en el 53,4\% de los casos.

- Por último, el cálculo para cinco años en meses es mayor que el de tres años en semanas en el $58,6 \%$ de los casos.

De manera lógica:

\section{$\mathrm{A}, \mathrm{B}>\mathrm{C}>\mathrm{D}$}

Donde:

$A=$ medida para un año en semanas

$\mathrm{B}=$ medida para un año en meses

$\mathrm{C}=$ medida para tres años en semanas

$\mathrm{D}=$ medida para cinco años en meses

Debido a que el cálculo semanal utiliza un número mayor de datos se elige a este como medida representativa: el cálculo del $\mathrm{R}^{2}$ medido en semanas a un año es el más representativo. 


\section{Referencias bibliográficas}

BOUCHET, Michel; SARMIENTO, Aleida y LUMBRERAS, José. 2007. El riesgo-país: un enfoque latinoamericano. Lima: ESAN.

ESTRADA, Javier. 2000. The Cost of Equity in Emerging Markets: A Downside Risk Approach. Barcelona: IESE, Departamento de Finanzas.

GARAY, Urbi y GONZÁLEZ Maximilano. 2005. Fundamentos de finanzas con aplicaciones al mercado venezolano. Caracas: IESA.
MONGRUT, Samuel y RAMÍREZ, Dídac. 2003. Discount Rates in Emerging Capital Markets. Lima: Universidad del Pacífico. Documento de Discusión.

MONGRUT, Samuel. 2006. Tasas de descuento en Latinoamérica: hechos y desafios. Lima: Universidad del Pacífico. Documento de Discusión.

SABAL, Jaime. 2002. Financial Decisions in Emerging Markets. Oxford: Oxford University Press. 